\title{
A methodology for preliminary sizing of a Thermal and Energy Management System for a hypersonic vehicle.
}

\author{
Roberta Fusaro ${ }^{1}$, Davide Ferretto ${ }^{1}$, Valeria Vercella ${ }^{1}$, Nicole Viola ${ }^{1}$, \\ Victor Fernandez Villace ${ }^{2}$ and Johan Steelant ${ }^{2}$
}

\begin{abstract}
This paper addresses a methodology to parametrically size thermal control subsystems for high-speed transportation systems. This methodology should be sufficiently general to be exploited for the derivation of Estimation Relationships (ERs) for geometrically sizing characteristics as well as mass, volume and power budgets both for active (turbopumps, turbines and compressors) and passive components (heat exchangers, tanks and pipes). Following this approach, ad-hoc semi-empirical models relating the geometrical sizing, mass, volume and power features of each component to operating conditions have been derived. As a specific case, a semi-empirical parametric model for turbopumps sizing is derived. In addition, the Thermal and Energy Management Subsystem (TEMS) for the LAPCAT MR2 vehicle is used as an example of a highly integrated multifunctional subsystem. The TEMS is based on the exploitation of liquid hydrogen boil-off in the cryogenic tanks generated by the heat load penetrating the aeroshell, all along the point-to-point hypersonic mission. Eventually, specific comments about the results will be provided together with suggestions for future improvements.
\end{abstract}

Keywords: Thermal and Energy Management System, sizing models, turbopumps, LAPCAT MR2

\section{Introduction}

The high operating temperature characterizing the hypersonic flight regime is a long-term issue. Vehicles able to reach hypersonic speed are currently considered for both aeronautical (e.g. high speed antipodal transportation systems) and space transportation purposes (e.g. reusable launcher stages and re-entry systems). In the past decades, several solutions have been envisaged to protect vehicles from high heat fluxes penetrating the aeroshell as well as to withstand and control significant heat loads resulting from the exposure to a high-temperature environment for a certain period of time. This generally led to the introduction of Thermal Protection Systems (TPS) and Thermal Control Systems (TCS) with peculiar features and functionalities depending on the specific applications.

In this context, the design and sizing of these on-board subsystems are a very challenging activity for engineers, especially during conceptual design activities. In particular, installation becomes an issue of primary importance when dealing with highly-integrated multifunctional subsystems. This is the case of the Thermal and Energy Management System (TEMS) to be integrated within the LAPCAT MR2 vehicle, a hypersonic cruiser project [1].

In detail, this paper works out a methodology for the development of semi-empirical models allowing components sizing on the basis of a set of operating conditions. The presented application to the TEMS relies on a subsystem architecture already presented in [1] and for which advanced studies concerning both the vehicle and the on-board systems [3] have already been published. Furthermore, this work provides a more complete understanding of the characteristics of TEMS and of its constituent components, paving the way for future studies of multi-functional systems for hypersonic vehicles.

After this short introduction, Section 2 briefly describes the existing estimation models that are available in literature, highlighting the reasons why they have been selected as reference documents but, at the same time, underlining the major issues to be overcome. Then, Section 3, describes the reference vehicle LAPCAT MR2 and provides some useful details on the envisaged point-to-point hypersonic mission and main operating points, together with some highlights on the TEMS architecture and components. Section 4 represents the core of the article providing a description of the suggested methodology and in parallel, Section 5 shows an example of the way in which this methodology can be applied to properly size turbopump assemblies. Results for the specific case of TEMS of the LAPCAT MR2 are presented in Section 6. Eventually, major conclusions and guidelines for future improvements are drawn.

\section{Existing Estimation Models}

Different design and sizing models focusing on components usually exploited in a Thermal and Energy Management Subsystem, or more generically in a Thermal Control System, are available in literature. In general, these sizing approaches are almost exclusively related to the definition of the components performance however with small contributions to the physical characterization of the components. This is a typical issue during the conceptual design

\footnotetext{
1 Politecnico di Torino, Corso Duca degli Abruzzi 24, 10129 - Turin (Italy), roberta.fusaro@polito.it, davide.ferretto@polito.it, valeria.vercella@polito.it,nicole.viola@polito.it

2 European Space Agency - ESTEC, Keplerlaan 1, 2201 AZ Noordwijk, (Netherlands), victor.fernandez.villace@esa.int, johan.steelant@esa.int
} 
phase where active components (such as turbopumps, turbines and compressors) are mainly described by their operating parameters and conditions. Conversely, passive components are usually characterized on the basis of their physical features (e.g. weight and thickness for tanks and pipes, etc....). This difference is mainly due to the fact that active components require a very detailed analysis upon the design of the operational envelope and related aspects. For example, this is the case for turbomachinery for which it is usual to refer to the analysis of the working cycle, the effects on fluid characteristics and induced pressure field as well as to general performance evaluation. During conceptual and preliminary design, it is actually necessary deriving those values to be used to easily and rapidly characterize the components in order to perform high-level trade-off analyses upon systems architecture. It is actually easier to associate numerical values to performance (often directly derived from high level requirements and constraints) rather than defining quantitative estimations for physical parameters. For physical characterization, it is usual to adopt qualitative correlations, leading the designer to the definition of components' classes. For example, the diameter of a turbomachinery is usually unknown during the conceptual design, but a rough idea of its sizing is given by the categorization of the component as axial, mixed or centrifugal flow.

It is also worth noticing that performance characteristics differ mainly from physical ones as the former can be computed by means of exact and closed mathematical formulations whilst physical aspects can only be hypothesized following best practices or general high-level requirements (usually depending on integration issues) acting as guidelines. Thus, it is quite common for active components, to compute performance directly, whilst physical characteristics are derived through statistical approaches or legacy data coming from literature analysis and/or reports resulting from test campaigns. The so-called Weight Estimation Relationships (WERs) are in fact equations formulating mass derivation strategies based upon specific operational parameters of the components collected as statistical population. This approach demonstrates some weaknesses, typically related to the quality of the components database upon which the statistical analysis is based. Even if it represents a good starting point to partially bridge the gap between physical and performance derivation, it still remains far from an exact derivation (actually it is an approximated result).

Another critical point of the approximated models is the aging of the available data. Almost the whole set of studies on this topic refer to aged report coming from space agencies (e.g. NASA) which faced this problem early in 1960's when the aerospace engineering domains knew its golden era. This may also affect the estimations, since coefficients and statistical drivers shall be subjected to actualization in order to provide correct results for modern components. It has to be noticed that this is one of the major showstoppers for highly innovative aerospace products with massive use of breakthrough innovative technologies. In these cases, the effect of aging is easier to be tackled, introducing or updating proper coefficients taking into account new production and manufacturing processes that may allow an increase in performance range. Moreover, regulation and certification policies are very useful guidelines for the definition of sizing algorithms.

In summary, considering this short digression over design approaches available in literature, it is possible to point out that:

- Performance and physical analyses are characterized by a different level of accuracy of the results, coming from exact / closed mathematical formulations and approximated estimations / qualitative hypotheses respectively.

- Physical estimations for active components available in literature are generally based upon statistical derivations, which are highly dependent on the technological characteristics of the historical period in which the population was built.

- Physical estimations for passive components are instead more reliable, since they are referring to structural resistance and strength required to operate within the limits prescribed by the environmental conditions (and by regulations / standards)

It shall be noticed that many references propose semi-empirical formulations for the derivation of physical characteristics of the main components starting from assumptions and models related to their performance. However, performance and physical worlds remain quite separated in the references since the output from one analysis is seldom used for the other. Starting from these considerations, the approach developed here aims at overcoming the limits of the statistical derivation, suggesting the development of proper semi-empirical models to increase the accuracy of formulations leading to the estimations of physical characteristics mass, dimensions and volumes, directly based upon operational parameters. The main difference with respect to the currently available methods is that, instead of using a database of components to build an Estimation Relationships (ERs), physical data are derived directly from operating parameters (using those exact performance relationships in a convenient way) and then compared to available values for validation purposes only. The introduction of coefficients and corrective parameters is adopted only for those configuration and fabrication characteristics that cannot be included in other ways. The approach has demonstrated a general validity even if higher attention has been devoted to the definition of estimation models for active components.

Focusing in particular on turbopumps characterization and design, a noticeable number of documents are available in literature. Apart from the pure theoretical derivation, available in different manuscripts, the attention has been focused on some technical reports [8][9][10]. These documents provide an overview of turbopump assemblies for liquid rocket engines together with an overview of the main parameters characterizing their performance. The 
turbopumps database included in the reports has been used as validation of the proposed model, since the data presented in the documents are related to both performance and physical characteristics of real turbopumps. However, since the data are referred to 1970's, the database has been enriched with more recent machines generally exploited for rocket space applications.

An interesting approach to the identification of physical characteristics of the machines is also provided in [11], where a theoretical approach for the estimation of some dimensionless parameters of turbomachines based on the Cordier diagram is suggested. The main governing equations presented in the paper have been a starting base of the proposed work and interesting relationships, relating the dimensionless parameters to each other, have been used as well. Concerning semi-empirical methods for the estimation of turbopumps mass and volumes, [12] has been selected as it provides a useful base for semi empirical predictive models, besides considering some very specific operational ranges. In this paper the authors propose the main equations for the determination of physical characteristics for a more general range of turbopumps by introducing some modifications to the original formulation presented in literature. Both relationships and their coefficients have been modified to be applicable to a wider spectrum of turbopumps and results have been compared to the original formulation for verification and validation purposes.

\section{Reference case Study: LAPCAT MR2 and the TEMS}

\subsection{LAPCAT MR2}

Besides targeting the derivation of a methodology for a wider applicability, also a real case study will be addressed, i.e. Thermal and Energy Management Subsystem for the LAPCAT MR2 vehicle. The LAPCAT MR2 is a hypersonic cruiser concept resulting from multiple design optimizations performed within the European Projects LAPCAT I and LAPCAT II [3]. The vehicle is characterized by a waverider configuration and it is equipped with six Air Turbo Rocket (ATR) [5][6] and one Dual Mode Ramjet (DMR)[4], which allow cruising Mach 8 at an altitude above $33000 \mathrm{~m}$, following the mission profile specified in Fig.1. The engines use liquid hydrogen (LH2) as fuel. A central intake, which is equipped with several moveable ramps [6], allows driving the airflow either to the ATR or to the DMR depending on the flight conditions.

The vehicle is conceived to host 300 passengers providing a commercial antipodal flight service across the globe and it is characterized by a Maximum Take-Off Weight (MTOW) of about 400 tons.

\subsection{Mission profile and Operating Conditions}

Notably, the six ATR operate up to Mach 4-4.5, whilst the DMR is used for hypersonic flight from Mach 4.5 up to Mach 8. The result is the mission profile reported in Fig. 1 [7], which is characterized by a first subsonic cruise, a supersonic ascent followed by the ATR switch-off and the ignition of the DMR and a subsequent hypersonic climb and cruise. The descent is then unpowered. The reference mission which has been taken into account for the vehicle is Brussel - Sidney direct route, characterized by a great circle distance of $16700 \mathrm{~km}$ and a flight time of about $2 \mathrm{~h} 45 \mathrm{~min}$.

Notwithstanding the fact that the cruise altitude is almost three times the flight level with respect to conventional aircraft, flying at this high speed causes higher heat fluxes on the vehicle shell and, consequently, higher temperature of the skin layers. For the selected range of Mach numbers, the temperature of the pressure side of the aeroshell may reach $1000-1200 \mathrm{~K}$ in the worst condition. This leads to the adoption of proper materials for the TPS, belonging particularly to ceramic family rather than metallic one, especially for Mach higher than 4 [2]. This is more critical for sharp appendices (wing leading edges, canard, nacelles...) and the lower part of the airframe (pressure side). However, flying at higher speed may be also an advantage not only considering overall mission time but also looking to the heat load accumulated by the vehicle indicated as the thermal paradox. It is in fact true that heat fluxes grow with increasing speed, but the heat has less time to penetrate the structure while the wall temperature doesn't increase proportionally with the total temperature. As example, Fig. 1 shows the different heat loads computation for a flight at Mach 5 and Mach 8 respectively [1].

Apart from the external balance, other strategies shall be considered to control the amount of heat flowing through the shell to internal compartment in order to guarantee proper survivability levels of both systems and payload. To face this problem, LAPCAT MR2 is also equipped with an innovative Thermal and Energy Management System (TEMS) which is conceived to use the boil-off vapours coming from the evaporation of the LH2 within the tanks as main cooling means for the cabin, the powerplant and the air-pack of the Environmental Control System. Moreover, the high-pressure liquid hydrogen is used to drive a dedicated turbine providing mechanical power to the devices of the TEMS itself, producing enough power margin for the other on-board subsystems. 

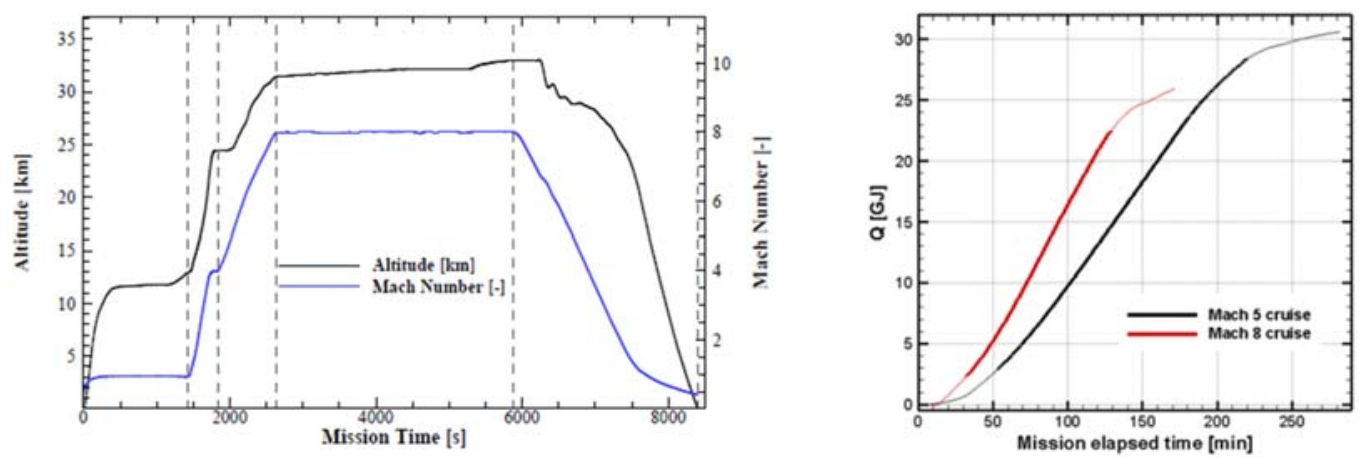

Fig. 1. Reference mission for LAPCAT MR2 and Integrated heat loads for different Mach [1]

\subsection{Thermal and Energy Management Subsystem}

The internal structure of the LAPCAT MR2 has been designed following a bubble-structure approach [14], leading to a peculiar integration of passenger compartment, fuel tanks and propulsion subsystems in a non-conventional architecture. In particular, Fig. 2 clearly shows the very high level of integration of the different compartments, maximizing the exploitation of available room for subsystem integration, in a sort of modular-approach [1].

This assembly is characterized by large interface surfaces between the cabin itself and the tanks containing LH2 at very low temperature (less than $20 \mathrm{~K}$ ), leading to strategic advantages. Indeed, with this configuration, the heat coming from the aerodynamic heating is conducted across the aeroshell to the tanks producing boil-off which can be used within a dedicated thermodynamic cycle. The so-called Thermal and Energy Management System (TEMS), sketched in Fig. 2 [1], makes benefit of the boil-off produced in LH2 tanks to cool down directly the passenger cabin. Moreover, the gaseous $\mathrm{H} 2$ is compressed and used as coolant within the air-pack of the ECS and the propulsion plant before the final injection within the combustion chamber.

The liquid $\mathrm{H} 2$ is pumped from the tanks to the engines, where it is used for cooling purposes and expanded within a dedicated turbine to produce mechanical power before mixing with the boil-off and injection in the combustion chamber. The turbine provides mechanical power to the devices of the TEMS and offers the possibility of using the remaining power to satisfy the needs of other on-board systems.

The main active components of the TEMS (pump, turbine and compressor) have already been preliminary sized in specific working conditions (design point) as reported in [15], providing the results shown in Table 1, where the power budget is also reported.
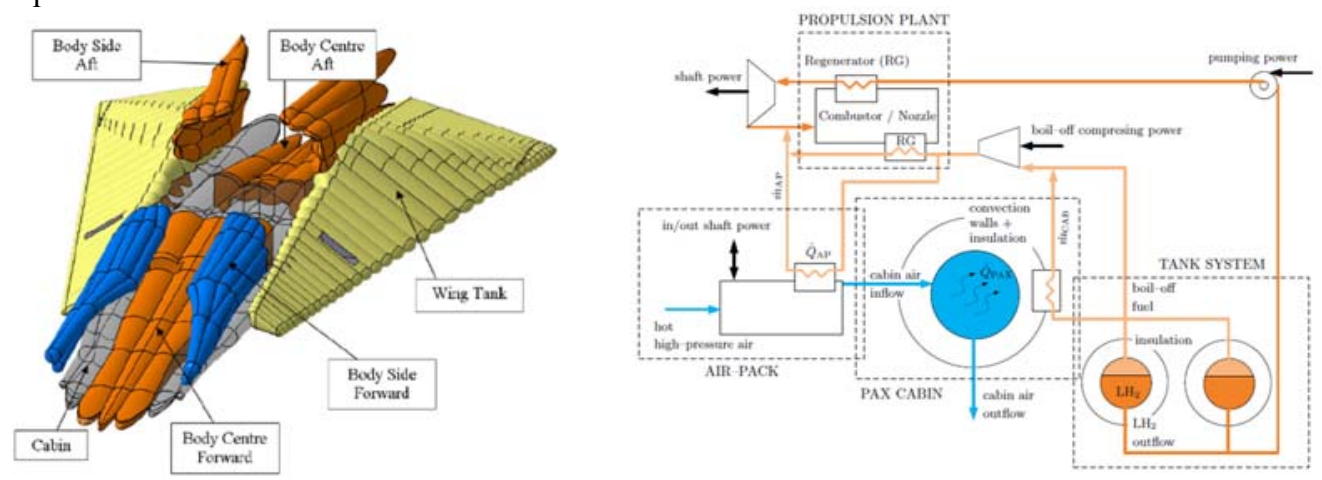

Fig. 2. Tanks and cabin layout of LAPCAT MR2 and scheme of the TEMS of LAPCAT MR2 [1]

Table 1. Design points of active components of the TEMS for LAPCAT MR2

\begin{tabular}{|l|c|c|c|c|c|c|}
\hline \multirow{2}{*}{$\begin{array}{c}\text { Component } \\
\text { Name }\end{array}$} & \multicolumn{2}{|c|}{ Temperature [K] } & \multicolumn{2}{c|}{ Pressure [bar] } & Mass flow & Power [MW] \\
\cline { 2 - 5 } & In & Out & In & Out & & \\
\hline Fuel Pump & 20 & {$[26-28]$} & 1 & {$[60-80]$} & {$[1-100]$} & {$[1-15]$} \\
\hline $\begin{array}{l}\text { Boil-off } \\
\text { compressor }\end{array}$ & {$[20-270]$} & {$[150-950]$} & 1 & 60 & {$[0-8]$} & {$[0-15]$} \\
\hline Turbine & 1300 & {$[1200-1300]$} & {$[60-80]$} & 60 & {$[10-100]$} & {$[0-120]$} \\
\hline
\end{tabular}




\section{Methodology}

\subsection{Methodology overview}

In view of the differences between performance and physical analyses, the derivation of correlations to estimate physical characteristics as function of a set of operational parameters and performance is challenging and demanding. Considering the complexity of this work and its multidisciplinary aspect, the exploitation of a systems engineering approach is proposed. This requires the focus moving progressively from the pure and limited technical engineering domain towards a multidisciplinary design. This is necessary to maximize the effectiveness of the entire vehicle rather than the efficiencies of each single component. In particular, the proposed approach aims at deriving a commonly integrated methodology that can be applied to a wide range of active and passive components in order to provide a reliable model for the prediction of their physical characteristics. Of course, each component should be analysed in detail and proper sizing algorithms elicited, but the methodology here presented suggests a unique rational of more general validity. Moreover, considering that these estimation relationships should be applicable as from the conceptual and preliminary design phases, each mathematical model shall be conceived to be easily integrated within the overall design approach, exploiting e.g. the output of performance analysis as main parameters for the mathematical formulations to predict mass, volume and dimensions of the devices. This will allow a standardization of the theoretical derivation approaches, the applicability to a wider range of components and a reduced impact on the workload. An overview of the overall derivation process is reported in Figure 3.

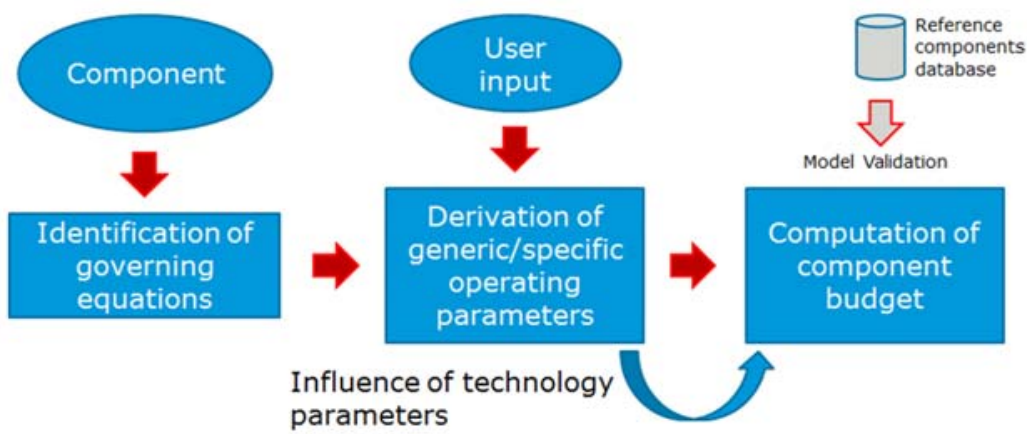

Fig. 3. Proposed derivation process

In particular, Fig. 3 reveals that the proposed methodology can be split into three different steps. The first one is related to the identification of the set of governing equations for the under-analysis component. This can be done by referring to the applicable scientific literature describing the behaviour of specific devices by constituting relationships based upon the main operating parameters. These parameters are the core of the methodology allowing the computation of the power budget. A proper classification of these parameters is performed within the second phase of the method, with the objective of analysing their impact on the governing equations. The identification of the relationships occurring among parameters and between variables and parameters, allows expressing each variable (e.g. mass, diameter, etc....) as a function of this set of parameters. In the third phase, through the exploitation of results coming from empirical estimations, simulations or literature, it is possible to find out the proper mathematical formulations to represent those relationships occurring between each variable and its subset of parameters.

Before entering in the detail of the methodology the following subsection aims at suggesting classification criteria for drivers.

\subsection{Preliminary drivers' classification}

In this context, different kinds of parameters or "drivers" can be identified depending on the variables they represent and on the physical phenomenon under evaluation. In particular, for the purposes of this study, four different categories have been identified:

- $\quad$ Generic Operating Parameters (GOP); they represent the main performance of a component (e.g. rotating speed of the machine, mass flow, pressure rise, efficiency, etc.). They are referred to as "generic" since different kinds of components can be described by means of them, as they describe the basic physical occurring phenomena.

- $\quad$ Specific Operating Parameters (SOP); differently from GOP, they are peculiar of a specific kind of machine (or at least of a family of machines). They can often be expressed in non-dimensional way to allow comparisons between similar components. They may assume also the aspect of simple coefficients conceived to represent the main characteristic of a machine with single value or through couples of variables (e.g. specific speed and specific diameter). 
- Secondary Parameters (SP); they are usually function of SOP and they can be related to the characteristics of subparts of the components (e.g. the inducer of a pump, the blade of a turbine etc....)

- Technology Parameters (TP); they are able to reflect the technology maturation level of a component. They may be expressed through coefficients and corrective factors, allowing a higher flexibility of the model (since they may be adopted to enlarge the limits of the correlations) even if causing a sort of discontinuity with the aim of the "exact formulation" of the estimations.

Eventually, when dealing with the development of these estimation models, the limited availability of data should be taken into account as it may impose a pruning of the parameters to be used in conceptual design phases. In fact, since the model is conceived to host a specific set of variables in its equations, it is important that the user may have access to these kinds of information, or it is necessary to provide the user with proper suggestions or formulations to estimate the missing data (e.g. it could be difficult to ask the user for a specific value of the tangential speed of the flow over an impeller blade, being easier to ask for general performance like the mass flow and then implementing a proper equation to derive it where it is necessary in the model). Thus, it is very important to define which subset of drivers among the GOP, the SOP and the SP shall be used to make the model applicable at the proper design level.

Then, the identified parameters are used in building up proper equations allowing the computation of the so-called system mass and volume budgets. Actually, power budgets may be computed as well since, in general for active components, the expression of power produced and consumed is directly related to their GOP. Moreover, the third phase is also the right place to perform the validation of the model, making benefit of the data available in literature concerning the physical characteristics of the components and comparing the results with the data coming from the real world. This can also lead to the modification of the correlations in case proper corrective trends are identified within the data.

Looking at the formal representation of the equations it is possible to say that, since the governing equations $G$ can be usually represented as in (1)

$$
G=g(G O P, S O P)
$$

the final estimation relationship $F$ will be assembled (2) as a function of the aforementioned drivers

where

$$
F=f(G, S P, T P)
$$

$$
S P=h(S O P)
$$

This brings us to the formalization of the estimation relationship $\mathcal{F}$ in the generic form:

$$
\mathcal{F}=f[g(G O P, S O P), h(S O P), T P]
$$

\section{Turbopumps}

This section aims at describing the step-by-step application of the generic methodology presented in Section 4, to the specific case of turbopump assemblies.

\subsection{Identification of governing equations (Step 1)}

Turbopumps are widely used devices for different aerospace applications, from engine feed (typically for rockets), to thermodynamics cycles that require moderate to high power. Different models for performance estimations are present in literature, starting from theoretical derivation of the operational parameters [8]. Moreover, some legacy data, referring to some specific design points, are available and can be used to statistically derive mass and sizing models [9] [12]. But unfortunately, as it has already been highlighted Section 2, pure statistical models are not applicable for the purposes of this paper as they are not establishing any relationships between physical characteristics and operating parameters.

However, an in-depth analysis of the existing literature is absolutely necessary to have a clear view of the wide spectrum of turbopump architectures that can be envisaged. A typical and useful characterization of these machines can be performed through the computation of two main parameters, the specific speed $N_{S}$ and the specific diameter $D_{s}$ respectively defined as in (5) and (6) [8].

where

$$
N_{s}=\frac{N Q^{1 / 2}}{H^{3 / 4}} \quad(5) \quad D_{s}=\frac{D H^{1 / 4}}{Q^{1 / 2}}
$$


$N$ is the rotating speed [rpm]

$Q$ is the volumetric flow rate (usually expressed in imperial units as [gpm])

$H$ is the head rise [ $\mathrm{ft}$ (as far as imperial units are concerned)

$D$ is the diameter of the machine [ $\mathrm{ft}$ (as far as imperial units are concerned)

Through these numbers, different machines can be compared directly on a single diagram, known as the Balje diagram, that represents a sort of map upon which many other coefficients can be plotted (as function of these two main parameters). The different types of turbopump architectures can be identified on the diagram, depending on the ranges of $N_{S}$ and $D_{s}$ (Fig. 4).

As example, the head and flow coefficients $\psi(7)$ and $\varphi(8)$ can be derived from the aforementioned numbers since

$$
\psi=\frac{g H}{u^{2}} \propto \frac{H}{N^{2} D^{2}}(7) \quad \varphi=\frac{v}{u} \propto \frac{Q}{N D^{3}}
$$

where

$g$ is the gravity constant $\left(9.81\left[\mathrm{~m} / \mathrm{s}^{2}\right]\right)$

$u$ is the peripheral velocity $[\mathrm{m} / \mathrm{s}]$

$v$ is the absolute velocity $[\mathrm{m} / \mathrm{s}]$

Please, notice that once head rise, the volumetric flow rate, the rotational speed of the machine and the characteristic velocities of the flow have been computed, the main data are already available for machine characterization, from the operational standpoint.

Considering that these values are strictly related to the operating conditions of the machine, a simplification of the design problem has been achieved [11] by looking at the definition of the optimum operating condition of a turbomachinery, working at $Q_{o p t}, \Delta p_{\text {opt }}, N_{\text {opt }}$ and optimum efficiency. In analogy with the definition of $N_{s}$ and $D_{s}$, similar constituting parameters, namely the speed $\sigma$ and diameter $\delta$ numbers $(9,10)$, and referring to a simpler diagram known as Cordier diagram (Fig. 4).

$$
\sigma=0,355 \Omega_{s} \quad(9) \quad \delta=\frac{\sqrt{\pi}}{2^{3 / 4}} D_{s}
$$

with

$$
\Omega_{s}=\frac{\Omega Q^{1 / 2}}{Y^{3 / 4}}
$$

where

$\Omega_{S}$ is the specific speed with reference to $\Omega$

$\Omega$ is the rotating speed $[\mathrm{rad} / \mathrm{s}]$

$Q$ is the volumetric flow rate $\left[\mathrm{m}^{3} / \mathrm{s}\right]$

$Y=g H$ is the specific head $[\mathrm{J} / \mathrm{kg}]$

Consequently, the expression of head and flow coefficients can be re-arranged as in (12) and (13).

$$
\psi=\frac{1}{2 \sigma^{2} \delta^{2}}(12) \quad \varphi=\frac{1}{\sigma \delta^{3}}(13)
$$

Equations (5) and (6) can be then identified as main governing laws for turbopumps and have the formal representation of

$$
\mathcal{G}=g(G O P)=g(\sigma, \delta)=g(N, Q, H, D)
$$



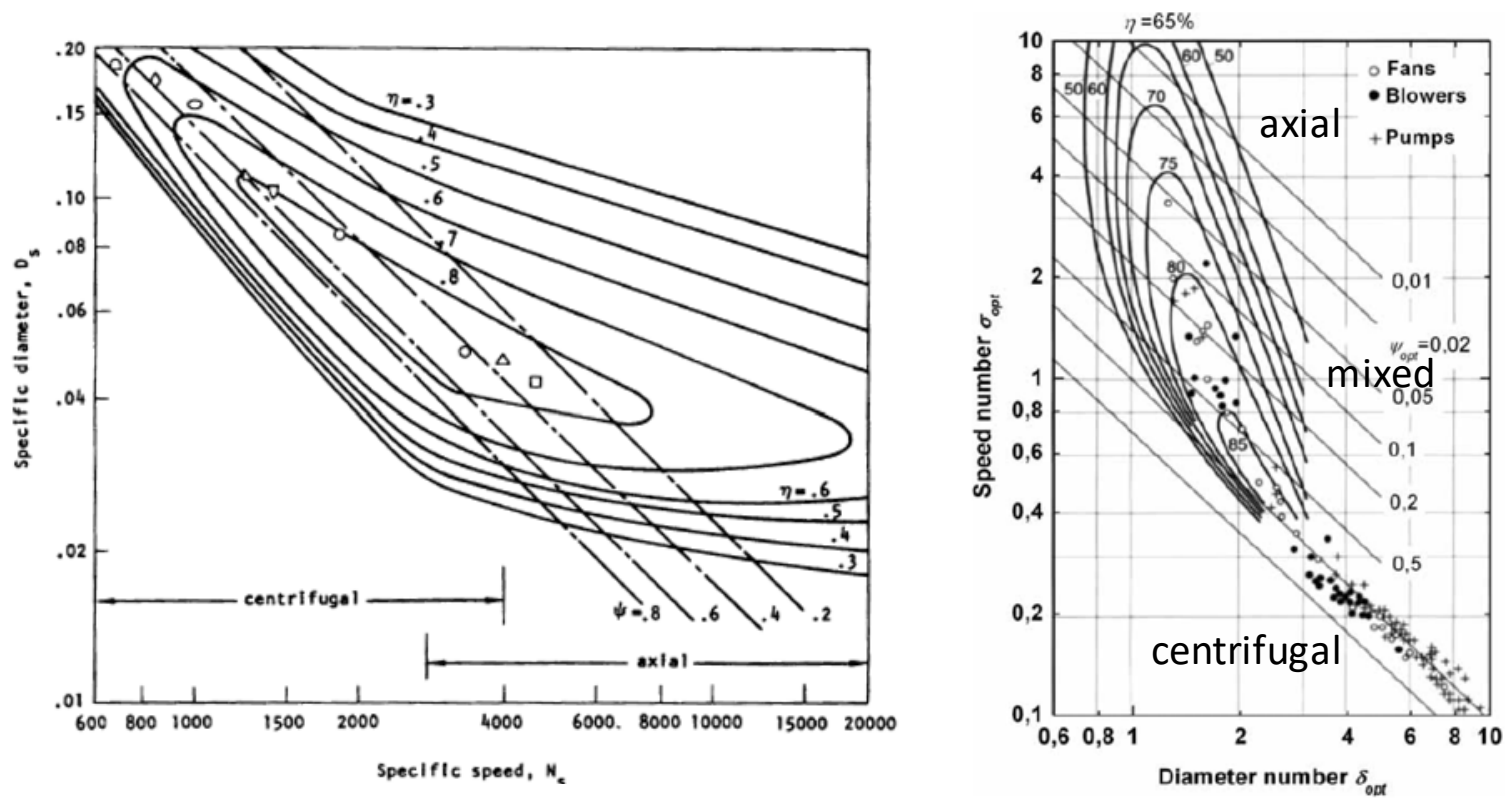

Fig. 4. Balje and Cordier diagrams for turbopumps

\subsection{Derivation of operating parameters (Step 2)}

In this section, the approach followed to derive the mathematical formulation of operating parameters characterizing turbopumps from governing equations is described. For the purposes of this work, the second group of governing equations is selected (9), (10) and re-arranged in order to be compatible with the hypotheses here described. The model described in [11] is taken as reference.

The volumetric flowrate $Q$, rotational speed $\Omega$ and head rise $H$ (or pressure rise $\Delta p$ / specific head $Y$ ) are supposed to be known by the user, since a first evaluation of the performance is required by the system. In this way, the computation of specific speed $\Omega_{s}$ and speed number $\sigma$ is straightforward as indicated in (9) and (11). Equation (10) can be used to derive the reference diameter as function of $\delta$ through the afore mentioned parameters (Fig. 5).

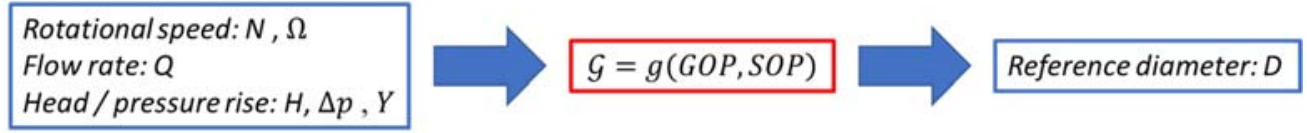

Figure 5. Variables flowchart for input / output of governing equation of turbopumps

However, $\delta$ is unknown as well at this stage. In order to overcome this problem, it is possible to make benefit of the equation which relates $\sigma$ and $\delta$ through the maximum efficiency of the machine $\eta_{\text {opt }}(15)$, which shall be hypothesized by the user. In fact, depending on the required efficiency, it is possible to derive the characteristic dimensions of the machine working within the previously specified operating conditions.

Imposing $\Delta=\delta^{2}$, Eq. (16) can be obtained:

$$
\eta_{\text {opt }}=1-\frac{1}{4 \delta^{2}}\left(\frac{4}{\delta^{2}}+\frac{1}{\sigma^{2}}\right)
$$

$$
4 \sigma^{2}+\Delta+4 \sigma^{2}\left(\eta_{o p t}-1\right) \Delta^{2}=0
$$

This is a second order algebraic equation which provides two families of results (17) (18):

$$
\Delta_{1}=\delta_{1,2}= \pm \sqrt{\frac{-1-\sqrt{1-64 \sigma^{4}\left(\eta_{o p t}-1\right)}}{8 \sigma^{2}\left(\eta_{o p t}-1\right)}} \quad \text { (17) } \quad \Delta_{2}=\delta_{3,4}= \pm \sqrt{\frac{-1+\sqrt{1-64 \sigma^{4}\left(\eta_{o p t}-1\right)}}{8 \sigma^{2}\left(\eta_{o p t}-1\right)}}
$$

Only positive values have physical relevance. Moreover, looking at the conditions of existence, it is possible to notice that the only possible solution is $\delta_{1}(19), \forall \sigma \neq 0$. 


$$
\delta_{1}=\sqrt{\frac{-1-\sqrt{1-64 \sigma^{4}\left(\eta_{o p t}-1\right)}}{8 \sigma^{2}\left(\eta_{o p t}-1\right)}}
$$

In this way, it is possible to obtain the formulation for the diameter, applying equations (10) and (6) in series. Moreover, with the derivation of $\delta$ it is also possible to compute head and flow coefficients $\psi, \varphi(7,8)$ and the subsequent flow velocities (7). In conclusion of this step, the governing equations for turbopumps can be obtained (20).

$$
\left\{\begin{array}{c}
\sigma=\frac{1}{\sqrt{\pi}} \frac{\Omega Q^{1 / 2}}{(2 Y)^{3 / 4}} \\
\eta_{o p t}=1-\frac{1}{4 \delta^{2}}\left(\frac{4}{\delta^{2}}+\frac{1}{\sigma^{2}}\right)
\end{array}\right.
$$

Once the machine is fully characterized, it is possible to move to the physical characterization of the turbopump assembly and related components, following the model proposed in [12]. Table 2 summarizes the main results of this methodology step. The different estimation relationships to derive turbopump dimensions and mass can be computed starting from these group of parameters.

\begin{tabular}{l|l}
\multicolumn{1}{l||}{ Parameter } & Type of parar \\
\hline \hline Volumetric mass flow $Q$ & GOP - Input \\
\hline Head rise (and related variables) $H$ & GOP - Input \\
\hline Rotational speed $N$ & GOP - Input \\
\hline Efficiency $\eta$ & GOP - Input \\
\hline Specific speed $\Omega_{S}$ & SOP \\
\hline Specific diameter $D_{S}$ & SOP \\
\hline Speed number $\sigma$ & SOP \\
\hline Diameter number $\delta$ & SOP \\
\hline Flow coefficient $\varphi$ & SOP \\
\hline Head coefficient $\psi$ & SOP \\
\hline Impeller blade angle $\beta$ & SP \\
\hline Absolute, relative and radial & SP \\
\hline velocities $c, u, w$ & \\
\hline
\end{tabular}

Table 2. List of operating parameters for turbopump governing equations

\subsection{Turbopump assembly arrangements}

This section aims at performing a review of the existing turbopump architectures with the final goal of understating in which way the different locations of the components affect the sizing predictive equations. Depending on the specific type of coupling between turbine and pumps, three different turbopump assemblies can be distinguished: direct driven, geared or dual shafts turbopumps.

Table 3. High level estimations strategies for the derivation of dimensions of different turbopump assemblies

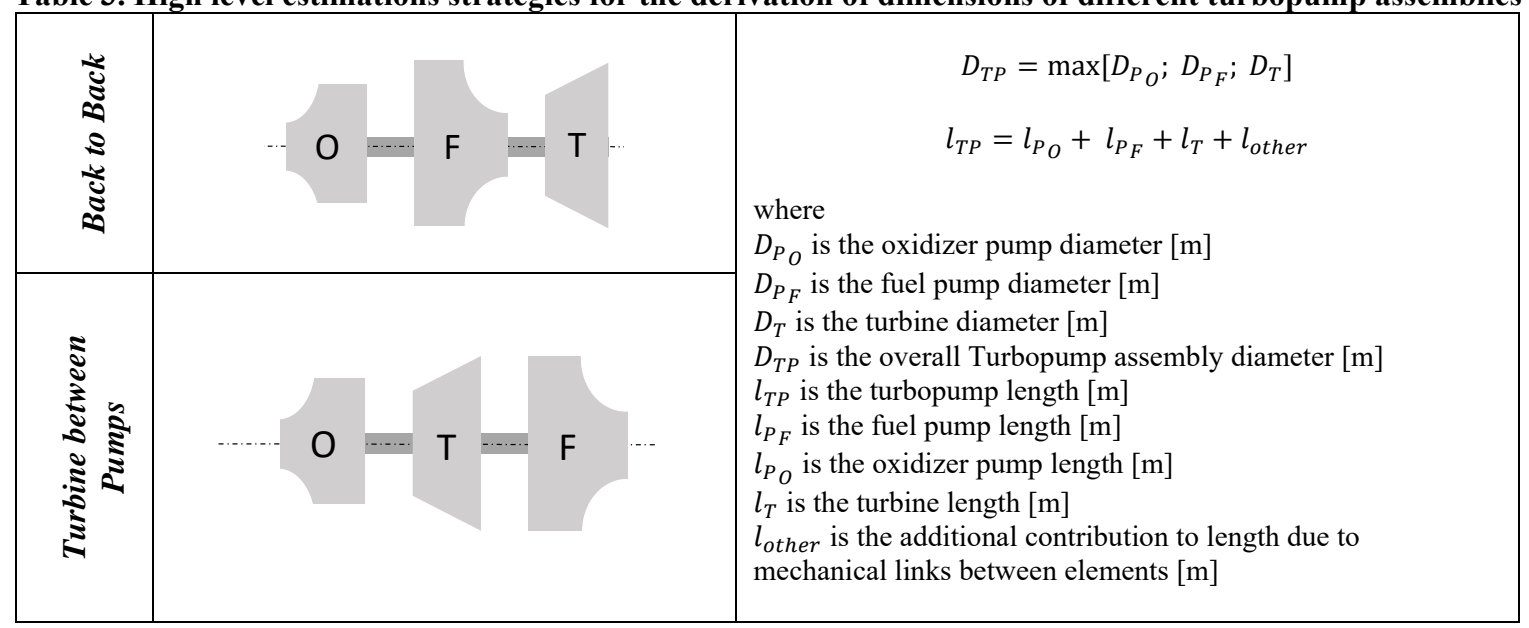




\begin{tabular}{|c|c|c|}
\hline 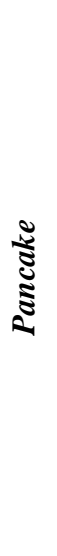 & & $\begin{array}{l}\qquad D_{T P}=\max \left(D_{P_{O}}+D_{P_{F}} ; \mathrm{D}_{\mathrm{G}}\right) \\
\qquad l_{T P}=\max \left(l_{P O} ; l_{P_{F}}\right)+l_{T}+l_{\text {other }} \\
\text { where } \\
D_{G} \text { is the diameter of the gear }[\mathrm{m}] \\
D_{T P} \text { is the overall Turbopump assembly diameter [m] } \\
D_{P_{O}} \text { is the oxidizer pump diameter [m] } \\
D_{P_{F}} \text { is the fuel pump diameter }[\mathrm{m}] \\
l_{T P} \text { is the turbopump length }[\mathrm{m}] \\
l_{P_{F}} \text { is the fuel pump length [m] } \\
l_{P_{O}} \text { is the oxidizer pump length [m] } \\
l_{T} \text { is the turbine length [m] } \\
l_{\text {other }} \text { is the additional contribution to length due to } \\
\text { mechanical links between elements [m] }\end{array}$ \\
\hline 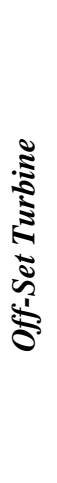 & $F$ & $\begin{array}{l}\qquad D_{T P}=\max \left(D_{P_{O}} ; D_{P_{F}}\right)+D_{T}-D_{G}^{\prime} \\
\qquad l_{T P}=l_{P_{O}}+l_{P_{F}}+l_{\text {other }} \\
\text { where } \\
D_{G}^{\prime} \text { is the percentage of overlapping [m] } \\
D_{T P} \text { is the overall Turbopump assembly diameter [m] } \\
D_{P_{O}} \text { is the oxidizer pump diameter [m] } \\
D_{P_{F}} \text { is the fuel pump diameter [m] } \\
l_{T P} \text { is the turbopump length [m] } \\
l_{P_{F}} \text { is the fuel pump length [m] } \\
l_{\text {other }} \text { is the additional contribution to length due to } \\
\text { mechanical links between elements [m] }\end{array}$ \\
\hline 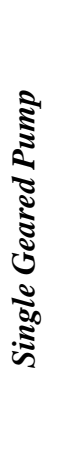 & $\cdots$ & $\begin{array}{l}\qquad D_{T P}=\max \left(D_{P_{O}}+D_{P_{F}} ; D_{G}\right) \\
\qquad l_{T P}=l_{P_{F}}+l_{T}+l_{\text {other }} \\
\text { where } \\
D_{G} \text { is the diameter of the gear }[\mathrm{m}] \\
D_{T P} \text { is the overall Turbopump assembly diameter [m] } \\
D_{P_{O}} \text { is the oxidizer pump diameter [m] } \\
D_{P_{F}} \text { is the fuel pump diameter [m] } \\
l_{T P} \text { is the turbopump length [m] } \\
l_{\text {other }} \text { is the additional contribution to length due to } \\
\text { mechanical links between elements [m] }\end{array}$ \\
\hline
\end{tabular}

As summarized in Table 3, when a single turbine directly drives both propellant pumps by means of a common shaft, two different configurations are possible. Indeed, the turbine can be located at the shaft end, in a back-to-back configuration or it can be simply placed between pumps. The first configuration is the most interesting for the applications dealt with in this paper. An example of this type of configuration is represented by the turbopump of the Rocketdyne F1 rocket, used on the Saturn V first stage. For both the configurations, the turbopump diameter will be driven by the larger diameter among the oxidizer pump, the fuel pump and the turbine. Considering the serial alignment of all the elements, the axial dimension of the overall assembly can be identified putting together their single length and adding a term to take into account for extra length due to element connections.

Considering Geared Turbopump arrangements, three different configurations can be envisaged, depending on the type of geared connections. The pancake type is applied in case of speed differentials between pumps and turbines and requires the exploitation of different reduction gears. Example of pancake configurations is the YLR87 - AJ-7 turbopump that was used on Titan launch vehicle. In case, since the two propellant pumps are rotating at the same speed but different with respect to the turbine, the gears should be used to connect the pumps shaft to the turbine shaft and this configuration is usually referred to as off-set turbine. An example of the off-set turbine configuration is the turbopump assembly of the MA-5 Booster stage. The last type of geared turbopump is the single-geared and it is used when the turbine can directly lead the fuel pump but gears are required to be placed in between the two pumps due to different 
rotating speeds. This configuration is present in RL10A-3-3 turbopump. As it is summarized in Table 3 both the diameter and the length of the assemblies are strongly affected by the arrangement. In particular, the Off-Set turbine configuration may be the most compact in terms of diameter.

Dual shafts configurations are widely used in aerospace, especially in rocket propulsion systems. For example, they have been used for the Space Shuttle Main Engine High Pressure Oxygen and Fuel Turbopumps (SSME - HPOTP and SSME - HPFTP), the Saturn V second stage J2 turbopumps and the more recent Vulcain, Vulcain II and Vinci Turbopumps, to be used on board Ariane 5 and Ariane 6. In this case, two different arrangements can be envisaged considering that depending on the way in which hot gases are fed into the turbine: series or parallel dual shafts. In this case, as it is summarized in Table 4, the diameter and length estimation should be performed separately for the two propellant turbopumps.

Table 4. High level estimation strategies for the derivation fo dimensions of dual shaft turbopumps

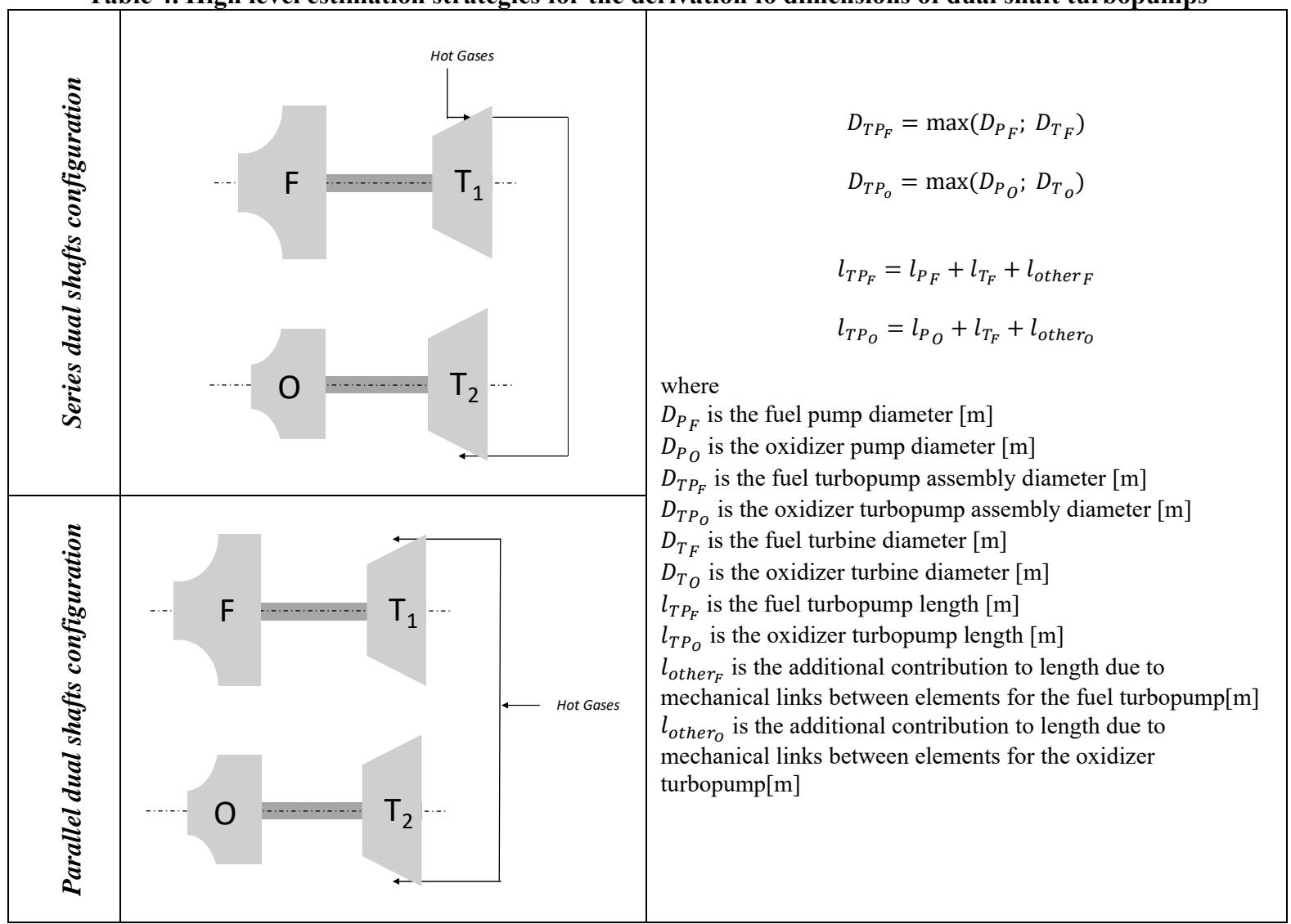

As example and in order to characterize the turbopumps within the database, Table 5 shows some existing turbopumps architecture with reference to the assemblies schematic reported in Table 4.

Table 5. Examples of existing turbopumps with reference to assemblies schematic

\begin{tabular}{|c|c|c|}
\hline $\begin{array}{c}\text { Turbopum } \\
\text { p name }\end{array}$ & Constructional Details & Assembly configuration \\
\hline \hline & & \\
SSME & &
\end{tabular}




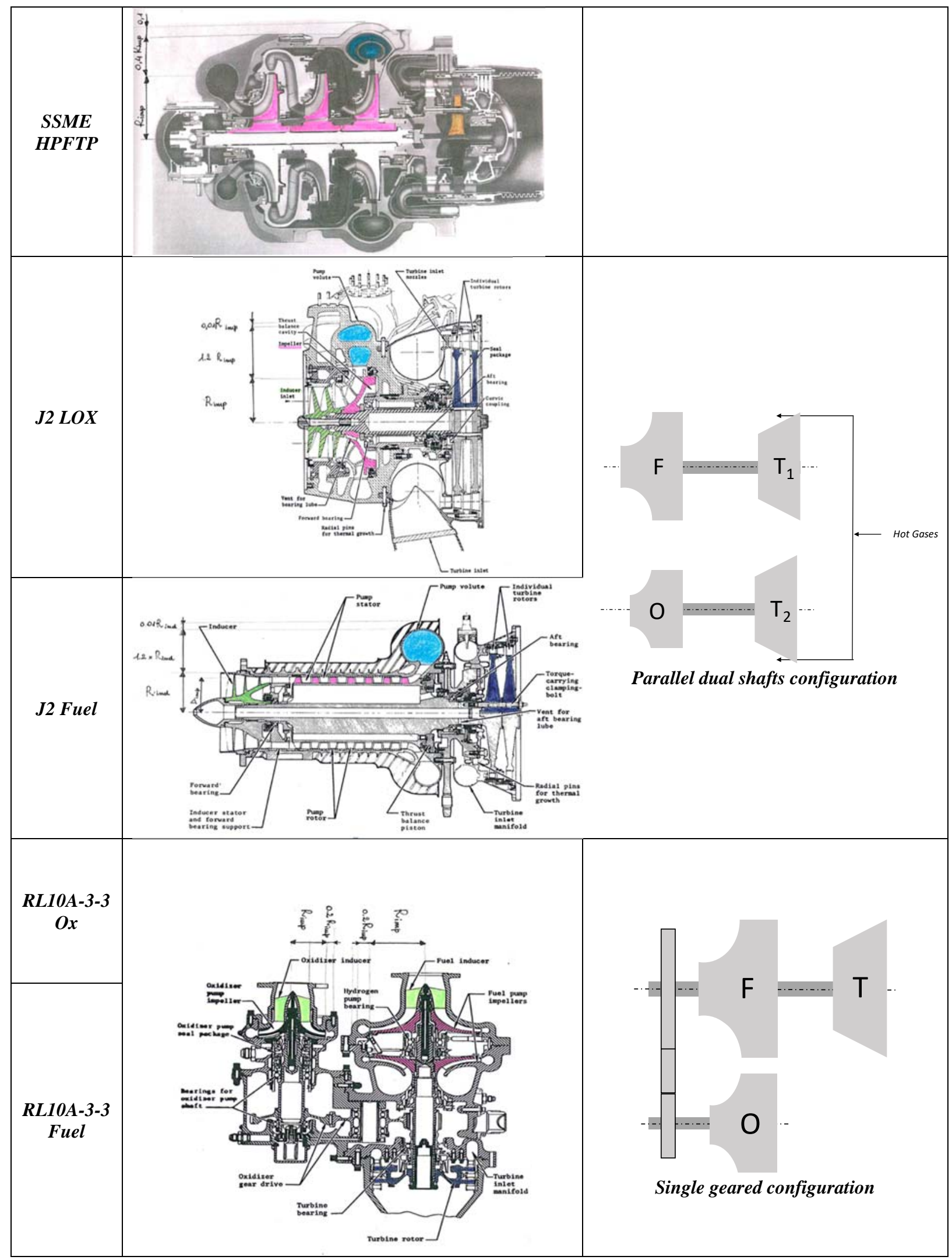




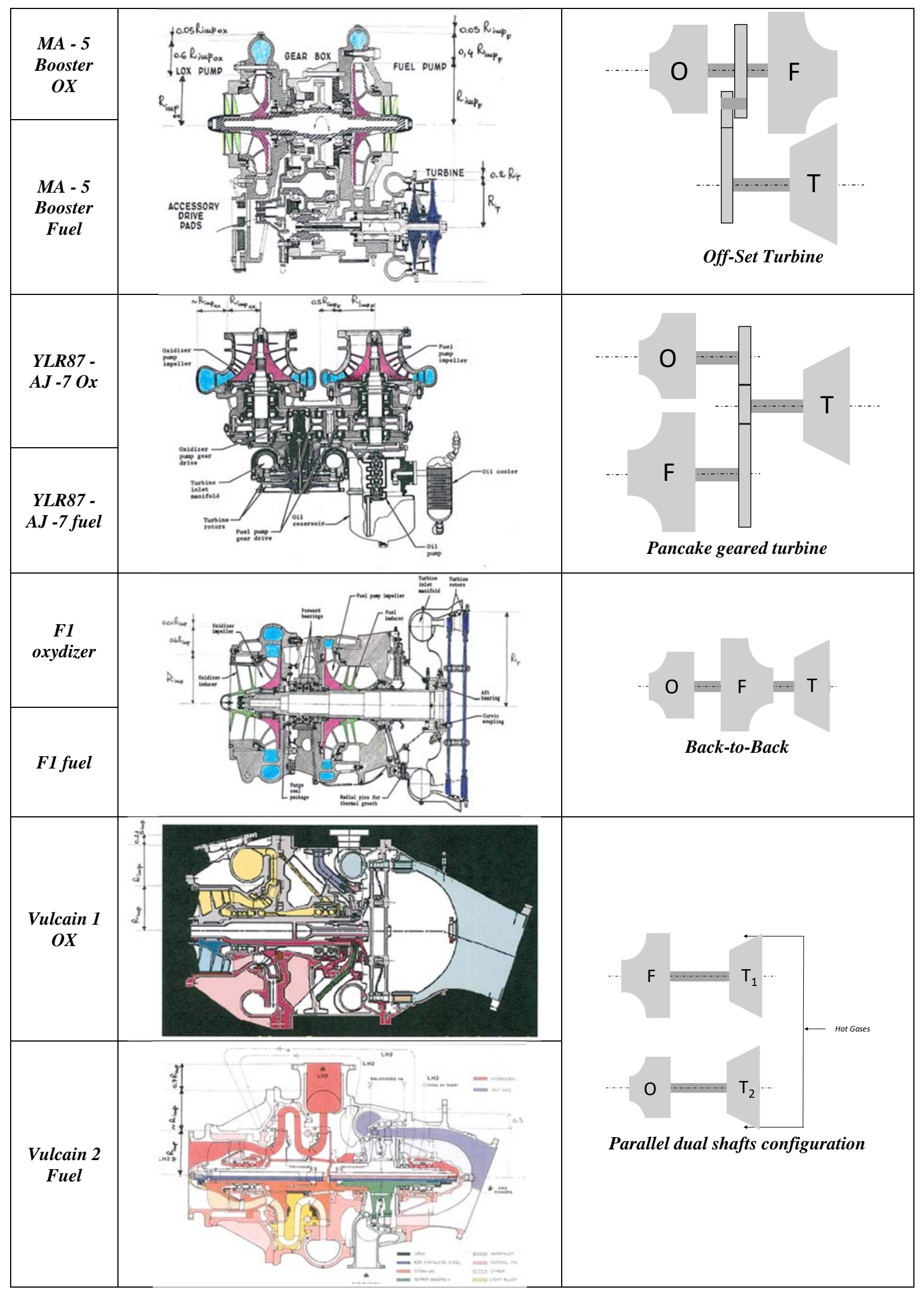




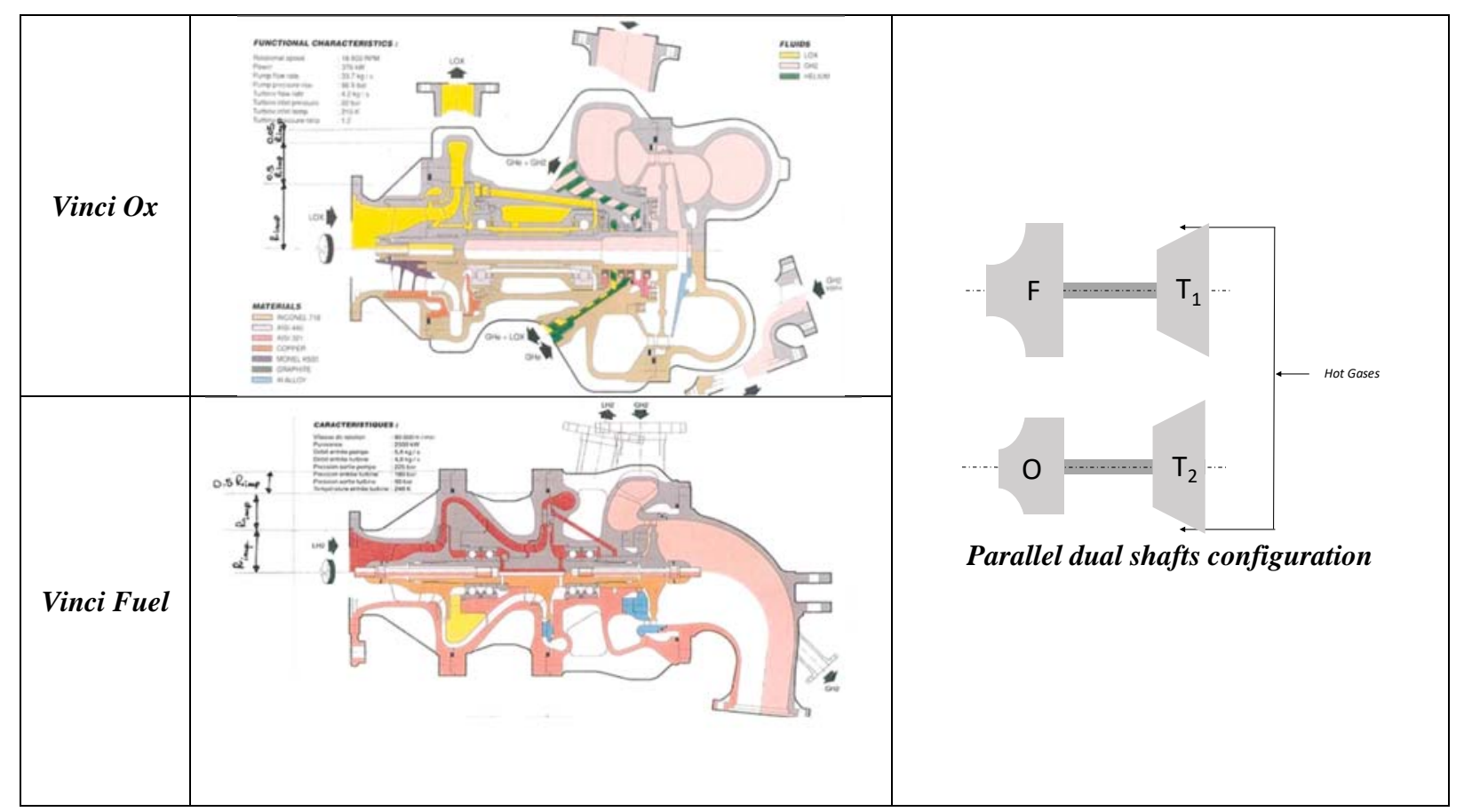

\subsection{Turbopump components sizing and budgets (Step 3)}

\subsubsection{Diameter Estimation (Model 1)}

The reference diameter is one of the most important physical parameters of the turbopumps when trying to characterize its volume and mass. This is due to the fact that, in general, the diameter of the machine can be related to the other physical features by means of semi-empirical models. In this section, useful semi-empirical formulations for the estimation of the diameter of the different components of a turbopump are reported. Considering the equations reported in the previous section, it is crystal clear that the components with a higher impact on the definition of the overall turbopump diameter are the pumps and turbines. However, in order to obtain more accurate results, especially in terms of mass budget, equations for the estimation of the other components are suggested too.

The dimeter of the pump component can be estimated considering the maximum between the impeller diameter and the inducer component. However, it has to be underlined that in all the analysed cases, the impeller contribution is always higher than the inducer one, when this last component is present.

The impeller diameter is directly related to the specific diameter reported in Eq. 6. But from the analysis of the various type of existing pumps, the inverse formulation (reported in Eq. 21) derived from Eq. 6 is only able to differentiate between machines with different operating fluids but it does not contain any information related to the impact of number of stages of the impeller, as well as of the impeller constructional type.

$$
D_{P_{\text {imp }}}{ }^{\prime}=\frac{D_{s} Q_{m}^{\frac{1}{2}}}{Y_{m}^{\frac{1}{4}}}
$$

Indeed, in case of multi-stages impellers, it shall be considered that they are mounted on the same shaft and, thus, they run at the same rotational speed. Moreover, since they are acting in series, they are supposed to handle the same fluid flow as an ideal single stage, with the same developed head. As a consequence, the impeller diameter of a multistage configuration should be smaller. Thus, the previous equation can be corrected making benefit of the affinity laws stating that:

- $\quad$ the pump volume flow rate varies directly with the rotational speed

- $\quad$ the pump-developed head varies directly as the square of speed

and assuming that the developed head developed by each stage of the impeller is equal to the head developed by the ideal single stage machine divided for the number of stages, the following equation (22) is suggested. 


$$
D_{P_{\text {imp }}}=\frac{D_{s} Q_{m}^{\frac{1}{2}}}{Y_{m}^{\frac{1}{4}}} \sqrt{\frac{1}{\left(n_{\text {stage }}\right)_{i m p}}}
$$

Considering the possible different configurations for each of these elements, the authors suggest to express all the missing contributions as proper percentages with respect to the impeller diameter, giving suggestions on how to tune these percentages depending on the characteristics of the element that is considered. It is worth noticing that the identification of proper factors representing the impact of the different components on the pump diameter has been based on an in-depth investigation of several components. Thus, the pump diameter can be estimated as in (23) and (24):

$$
\begin{gathered}
D_{P}=D_{P_{\text {imp }}}\left(1+k_{P_{\frac{D}{\text { other }}}}\right) \\
k_{P_{D / \text { other }}}=k_{D / \text { diff }}+k_{D / \text { inlet }}+k_{D / \text { outlet }}+k_{D / \text { misc }}+k_{D / \text { integration }}
\end{gathered}
$$

where

$k_{D / \text { diff }}$ is the contribution of the diffuser to the diameter estimation (see Table 5);

$k_{D / \text { inlet }}$ is the contribution of the inlet to the diameter estimation;

$k_{D / \text { outlet }}$ is the contribution of the inlet to the diameter estimation;

$k_{D / m i s c}$ is the contribution of other elements (connectors, etc..) to the diameter estimation (this value is strictly related to the under-design turbopump);

$k_{D / \text { integration }}$ is the contribution of components integration (e.g. casing, insulation, etc..) to the estimation (a value of 0.3 is suggested);

The impact of the diffuser onto the diameter estimation can varies from 0.1 to 1.2 and it depends on the type of geometry adopted to build the diffuser vane. Indeed, it has to be mentioned that the diffuser aim is to convert the velocity head in pressure head with as little turbulence as possible. This is done in one or more passages surrounding the impeller. Three major types of impeller might be identified:

- $\quad$ annular diffuser $\left(k_{D / \text { diff }}\right.$ lower that 0.5$)$

- $\quad$ volute diffuser $\left(k_{D / \text { diff }}\right.$ lower than 0.8$)$

- diffusing vane $\left(k_{D / \text { diff }}\right.$ higher than 0.8$)$

The impact of inlet and outlet vanes, as well as of the other secondary elements is strictly related to the geometry of the turbopump assembly. However, typical numbers, for different configurations, are reported in Table 6.

Please, notice that the constructional architecture of the impeller, is not affecting the diameter, while it is considered in the next section because it deeply affects the length of the pump.

\subsubsection{Diameter Estimation (Model 2)}

In literature, other interesting models have already been presented. In particular, the model presented by the Royal Air Force [12] has been considered interesting and a good additional reference model to compare the proposed results and validate it.

Following the suggestions reported in the RAF model, the diameter of the pump can be evaluated starting from the consideration that the delivery pressure of the pump can be estimated as the sum of two different contributions: the dynamic and the static pressure.

$$
p=\frac{1}{2 g} \rho\left(U^{2}-u^{2}\right)+\frac{\psi \rho U^{2}}{2 g}
$$

where

$U$ is the speed at the tip of the pump impeller; $u$ is the speed at the impeller hub. 
$\psi$ pressure recovery factor ( 0.2 suggested value [12],);

$U$ is the peripheral velocity at the outer radius of the impeller [ft/s];

$u$ is the peripheral velocity at the hub of the impeller [ft/s];

$g$ is the gravity constant $\left(32.18\left[\mathrm{ft} / \mathrm{s}^{2}\right]\right)$.

Considering that the two speeds can be easily put in relationship with the radius at the hub or tip section of the impeller blade (see Eq. 26 and 27), and remembering that the radius at the but can be expressed by Eq. 28,

$$
\begin{gathered}
u=2 \pi r_{\text {impeller }} n \\
U=2 \pi R_{\text {impeller }} n
\end{gathered}
$$

and that the radius at the hub can be expressed as:

$$
r_{\text {impeller }}=\sqrt{\frac{\dot{m}}{\rho \pi u}}
$$

Eq. (29) expresses the external radius estimation equation:

$$
R_{\text {impeller }}=\sqrt{C_{i_{1}} \frac{p}{n^{2} \rho}+C_{i_{2}} \frac{\dot{m}}{\rho u}}
$$

Thus, comparing Eq. (22) to Eq. (29), it is possible to understand the meaning of the $C_{i 1}$ and $C_{i 2}$ coefficients in the original formulation.

$$
\begin{aligned}
C_{i 1} & =\frac{g}{2 \pi^{2}(1+\psi)}\left[\frac{f t}{s^{2}}\right] \\
C_{i 2} & =\frac{1}{\pi(1+\psi)}
\end{aligned}
$$

However, this formulation, like the one suggested in [12] does not allow to take into account the effect of the number of stages of the impeller. Thus, the overall pump formulation will also include the correction due to the number of stages. The additional contributions, mainly due to the presence and impact of the other components are evaluated following the same suggestions implemented in Eq. 32.

$$
D_{P}=2 R_{\text {impeller }}\left(1+k_{P \frac{D}{\text { other }}}\right)
$$

The following table reports the major results related to the application of both models for the estimation of the diameter of some existing turbopumps developed for aerospace propulsion systems. As it is possible to notice, besides these two models starts with different hypotheses, they are both able estimating diameter values very closed to the real data available from literature.

Table 6. Coefficient used for turbopump diameter estimation and results obtained using the different models

\begin{tabular}{|c|l|l||l|l|l|l||l||l|l|}
\hline $\begin{array}{c}\text { Turbopump } \\
\text { name }\end{array}$ & Flow type & $\begin{array}{c}\text { Impeller } \\
\text { stages }\end{array}$ & $\boldsymbol{k}_{\text {D/diff }}$ & $\boldsymbol{k}_{\text {D/inlet }}$ & $\boldsymbol{k}_{\text {D/outlet }}$ & $\boldsymbol{k}_{\text {D/misc }}$ & $\begin{array}{c}\text { Diameter } \\
\text { Real [m] }\end{array}$ & $\begin{array}{c}\text { Model 1 } \\
\text { [m] }\end{array}$ & $\begin{array}{c}\text { Model 2 } \\
\text { [m] }\end{array}$ \\
\hline $\begin{array}{c}\text { SSME } \\
\text { HPOTP }\end{array}$ & Radial & 1 & 0 & 0 & 0 & 2.5 & 0.39 & 0.374 & 0.3812 \\
\hline $\begin{array}{c}\text { SSME } \\
\text { HPFTP }\end{array}$ & Radial & 3 & 0.3 & 0 & 0 & 0.1 & 0.55 & 0.54 & 0.5498 \\
\hline J2 LOX & Radial & 1 & 1.2 & 0 & 0 & 0.01 & N/A & 0.7 & 0.5822 \\
\hline J2 Fuel & Axial & 7 & 1.2 & 0 & 0 & 0.01 & N/A & 0.31 & 0.3002 \\
\hline $\begin{array}{c}\text { RL10A-3-3 } \\
\text { Ox }\end{array}$ & Radial & 1 & 0.2 & 0 & 0 & 0.01 & N/A & 0.25 & 0.1882 \\
\hline $\begin{array}{c}\text { RL10A-3-3 } \\
\text { Fuel }\end{array}$ & Radial & 2 & 0.2 & 0 & 0 & 0.03 & N/A & 0.29 & 0.2762 \\
\hline $\begin{array}{c}\text { MA - 5 } \\
\text { Booster OX }\end{array}$ & Centrifugal & 1 & 0.6 & 0 & 0 & 0.05 & N/A & 0.246 & 0.554 \\
\hline MA - 5 & Centrifugal & 1 & 0.4 & 0 & 0 & 0.05 & N/A & 0.32 \\
\hline
\end{tabular}




\begin{tabular}{|c|c|c|c|c|c|c|c|c|c|}
\hline $\begin{array}{c}\text { Booster } \\
\text { Fuel }\end{array}$ & & & & & & & & & \\
\hline $\begin{array}{c}\text { YLR87 - AJ } \\
-7 \text { Ox }\end{array}$ & Centrifugal & 1 & 1 & 0 & 0 & 0.01 & N/A & 0.48 & 0,51 \\
\hline $\begin{array}{c}\text { YLR87 - AJ } \\
-7 \text { fuel }\end{array}$ & Centrifugal & 1 & 0.7 & 0 & 0 & 0.01 & N/A & 0.53 & 0.55 \\
\hline F1 oxydizer & Centrifugal & 1 & 0.6 & 0 & 0 & 0.01 & N/A & 0.91 & 0.8433 \\
\hline F1 fuel & Centrifugal & 1 & 0.1 & 0 & 0 & 0.01 & N/A & 0.83 & 0.797 \\
\hline $\begin{array}{c}\text { Vulcain } 1 \\
\text { OX }\end{array}$ & Centrifugal & 1 & 0.7 & 0,2 & 0 & 0.01 & 0.5 & 0.45 & 0.4542 \\
\hline $\begin{array}{c}\text { Vulcain } 2 \\
\text { Fuel }\end{array}$ & Centrifugal & 2 & 1 & 0 & 0.7 & 0.01 & N/A & 0.55 & 0.5603 \\
\hline Vinci $O x$ & Centrifugal & 1 & 0.9 & 0 & 0 & 0.05 & N/A & 0.37 & 0.3741 \\
\hline Vinci Fuel & Centrifugal & 2 & 1 & 0 & 0 & 0 & 0.25 & 0.25 & 0.2533 \\
\hline
\end{tabular}

In addition, the diameter of the different subparts can be evaluated as follows (33 to 36).

$$
\begin{gathered}
D_{T}=\frac{k_{2}}{n} \\
D_{B}=2 D_{\text {Shaft }} \\
D_{P}=2 k_{3} R_{\text {impeller }} \\
D_{S}=2 D_{\text {Shaft }}
\end{gathered}
$$

where

$k_{2}$ and $k_{3}$ are constants

\subsubsection{Length Estimation}

A similar approach is here suggested to evaluate the overall Length of the turbopump on the basis of its constituent components. In particular, considering the different possible assembly configurations, turbopump overall length can be estimated considering the formulations reported in Table 7. This section aims at entering in the details of the different element contributions to the overall turbopump weight.

In the most generic case, where all the components are present, the turbopump length (37) can be evaluated as the sum of several contributes:

where

$$
l_{T P}=l_{\text {imp }}+l_{p b}+l_{\text {ind }}+l_{b}+l_{t}+l_{\text {out }}+l_{\text {other }}
$$

$l_{\text {imp }}$ is the impeller length at the hub;

$l_{p b}$ is the length of the pre-burner that might be present (usually expressed as a percentage with respect to $l_{i m p}$ );

$l_{\text {ind }}$ is the length of the inducer;

$l_{b}$ is the length of the bearings;

$l_{t}$ is the length of the turbine;

$l_{\text {out }}$ is the length of the outlet section of the turbine.

The length of the impeller can be usually estimated on the basis of the mass flow that the pump should handle and remembering that the length of the impeller is in proportion with the inlet pump diameter (38):

$$
l_{i m p}=\frac{r_{i n}}{2}
$$

where the inlet radius $\left(r_{i n}\right)$ can be estimated as it follows (39)

$$
r_{\text {in }}=\sqrt{\frac{\dot{m}}{\rho u \pi}}
$$


Again, this formulation does not allow to consider multi-stages impellers and does not reflect the type of impeller blade. Thus, the authors suggest to evaluate $l_{\text {imp }}$ through Eq. (40).

$$
l_{\text {imp }}=k_{\text {imp }} n_{\text {stages }} \sqrt{\frac{\dot{m}}{\rho u \pi}}
$$

where $k_{i m p}$ is a coefficient that takes into account the blade shape. In case of pure radial impeller, $k_{\text {imp }}=0.7$ is suggested while moving to mixed flow types towards Francis construction, higher values should be adopted (1.2 mixed flow and 2 for Francis type).

The other elements contributions are usually estimated as percentage with respect to the impeller length, apart from bearings and turbines, for which equations coming from some best practices may be used (see Eq. 41)

$$
\begin{aligned}
l_{b}=2 D_{\text {shaft }} & =2 \frac{u_{\text {shaft }}}{\left(\pi n_{T}\right)}=2 \frac{0.1 u_{T}}{\left(\pi n_{T}\right)} \\
l_{t} & =0.083 n_{\text {stages }}[f t]
\end{aligned}
$$

where

$D_{\text {shaft }}$ is the diameter of the rotating shaft $[\mathrm{ft}]$

$u_{T}$ tubine speed at the blade tip [m/s];

$n_{T}$ is the rotating speed of the turbine [rpm]

Eventually, the turbopump length (37) can be expressed as follows:

$$
l_{T P}=l_{\text {imp }}\left(1+k_{\frac{L}{\text { ind }}}+k_{\frac{L}{p b}}+k_{\frac{L}{\text { out }}}+k_{\frac{L}{\text { other }}}\right)+l_{b}+l_{t}
$$

\begin{tabular}{|c|c|c|c|c|c|c|}
\hline \multirow[b]{2}{*}{ Turbopump name } & \multicolumn{4}{|c|}{ Input data } & \multicolumn{2}{|c|}{ Results } \\
\hline & $k_{L / \text { ind }}$ & $k_{L / p b}$ & $k_{L / o u t}$ & $\boldsymbol{k}_{L / \text { other }}$ & $\begin{array}{c}\text { Real Length } \\
{[\mathrm{m}]}\end{array}$ & $\begin{array}{c}\text { Estimated } \\
\text { Length [m] }\end{array}$ \\
\hline SSME HPOTP & 2 & 1 & 0 & 4 & 0.805 & 0.81 \\
\hline SSME HPFTP & 0.5 & 0.5 & 0.7 & 1 & 0.96 & 1.05 \\
\hline $\mathrm{J} 2 \mathrm{LOX}$ & 2 & 0 & 0 & 5.5 & N/A & 0.73 \\
\hline J2 Fuel & 0.3 & 0 & 0 & 0 & $\mathrm{~N} / \mathrm{A}$ & 0.84 \\
\hline RL10A-3-3 Ox & 2 & 0 & 0 & 6 & $\mathrm{~N} / \mathrm{A}$ & 0.23 \\
\hline RL10A-3-3 Fuel & 0.8 & 0 & 0 & 2 & $\mathrm{~N} / \mathrm{A}$ & 0.38 \\
\hline MA - 5 Booster OX & 1 & 0 & 0 & 1 & $\mathrm{~N} / \mathrm{A}$ & 0.42 \\
\hline MA - 5 Booster Fuel & 1 & 0 & 0 & 1 & N/A & 0.37 \\
\hline YLR87 - AJ -7 Ox & 0 & 0 & 0 & 2.5 & N/A & 0.51 \\
\hline YLR87 - AJ -7 fuel & 0 & 0 & 0 & 1.5 & $\mathrm{~N} / \mathrm{A}$ & 0.42 \\
\hline F1 oxydizer & 1 & 0 & 0 & 1 & $\mathrm{~N} / \mathrm{A}$ & 0.6 \\
\hline F1 fuel & 0 & 0 & 0 & 4 & N/A & 0.92 \\
\hline Vulcain 1 OX & 2.5 & 0 & 3.5 & 4.5 & 0.7 & 0.63 \\
\hline Vulcain 2 Fuel & 0 & 0 & 0 & 6 & N/A & 0.715 \\
\hline Vinci $O x$ & 3 & 0 & 0 & 10 & N/A & 0.4 \\
\hline Vinci Fuel & 0.2 & 0 & 1 & 0.2 & 0.4 & 0.41 \\
\hline
\end{tabular}

This model has been applied to the same set of turbopump assemblies and the input and results are collected in Table 7

Table 7. Coefficients used for turbopump length computation and related results

\subsubsection{Mass, volume and power budgets estimation}

Then, a high-level estimation of the volume can be executed by simply multiplying the diameter of the machine and its length (45). 


$$
\begin{gathered}
V_{p}=\frac{l_{P} \pi D^{2}}{4} \\
M_{T P}=I\left(M_{P_{\text {casing }}}+M_{P_{\text {impeller }}}+M_{T_{\text {rotor }}}+M_{T_{\text {shaft }}}+M_{B}\right)
\end{gathered}
$$

The masses of pump casing (46) and impeller (47) as well as the turbine mass can be derived making benefits of similar considerations.

$$
\begin{gathered}
M_{P_{\text {casing }}}=\rho_{c P}\left(\pi R_{\text {impeller }}^{2}\right) l_{P} \\
M_{P_{\text {impeller }}}=\rho_{P} R_{\text {impeller }}^{2} b_{\text {impeller }} \\
M_{\text {rotor }}=l_{T} R_{T}^{2} \rho_{r}
\end{gathered}
$$

In particular, considering the results of the validation process and a subsequent sensitivity analysis, the coefficient has been expressed as

$$
I=I_{N} \cdot I_{\dot{m}}
$$

Suggested values for these coefficients are reported in Table 8 and 9.

\section{Table 8. Values of $I_{N}$ coefficient}

\begin{tabular}{c||l} 
Rotational Speed [rpm] & $\boldsymbol{I}_{\boldsymbol{N}}$ value \\
\hline \hline$N \leq 8,000 \mathrm{rpm}$ & 0.17 \\
\hline $8,000<N \leq 10,000 \mathrm{rpm}$ & 0.25 \\
\hline $10,000<N \leq 20,000 \mathrm{rpm}$ & 0.5 \\
\hline $20,000<N \leq 30,000 \mathrm{rpm}$ & 0.55 \\
\hline $30,000<N \leq 80,000 \mathrm{rpm}$ & 0.6 \\
\hline $80,000 \mathrm{rpm}<N$ & 0.75 \\
\hline
\end{tabular}

Table 9. Values of $I_{\dot{m}}$ coefficient

\begin{tabular}{c||l} 
Mass Flow rate $[\mathrm{kg} / \mathrm{s}]$ & $\boldsymbol{I}_{\dot{\boldsymbol{m}}}$ value \\
\hline \hline$\dot{m} \leq 100 \mathrm{~kg} / \mathrm{s}$ & 1 \\
\hline $100<\dot{m} \leq 300 \mathrm{~kg} / \mathrm{s}$ & $1.5-2^{*}$ \\
\hline$\dot{m}>300 \mathrm{~kg} / \mathrm{s}$ & 5 \\
\hline
\end{tabular}

*this range of values is due to the impact of pressure rise. Lower $I_{\dot{m}}$ values are suggested for low values of $\Delta p$.

$I$ and $k_{1}^{\prime}$ can be grouped as those technology parameters (TP) that can be identified for turbopump case study.

Eventually, power budget for the overall turbopump can be evaluated with the simple equation (50), where the power required is the product of volumetric flow rate and delivery pressure with an additional efficiency.

$$
P_{T P}=\frac{p Q}{\eta_{P}}
$$




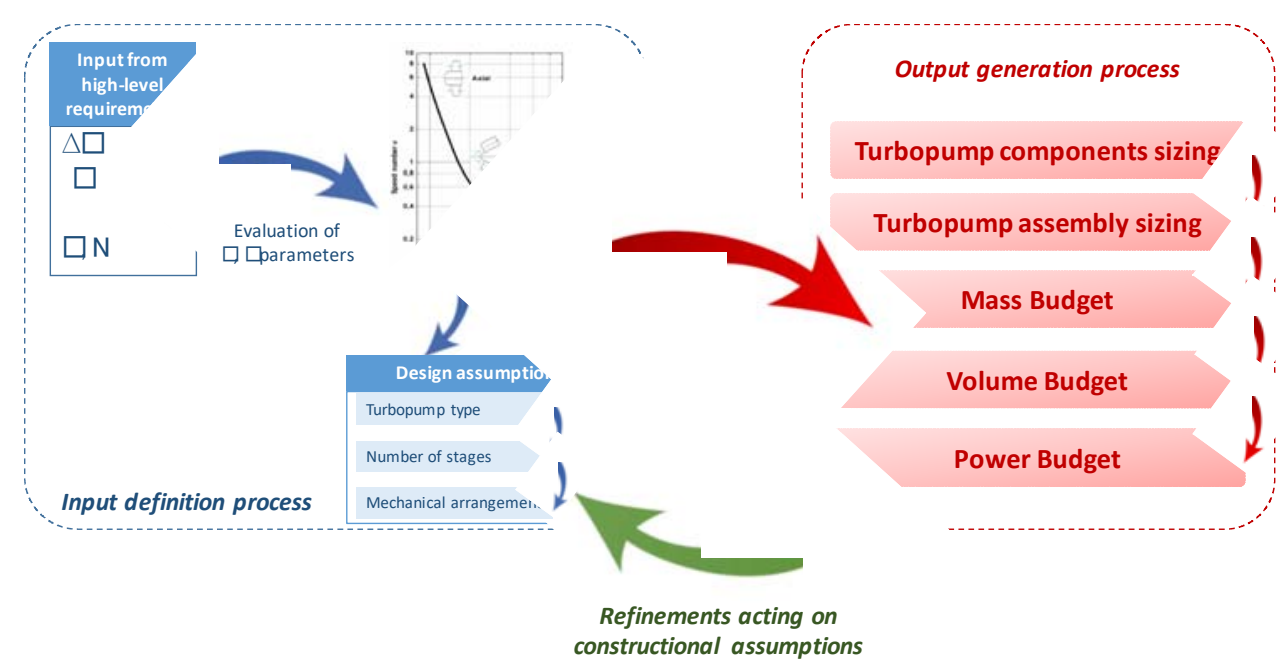

Fig. 6. Activity flow-chart to summarize the input-output definition processes

\subsection{Validation of the model}

Table 10 contains the database of the turbopumps used for the validation of the models presented in Section 5.4. Unfortunately, only a subset of these turbopups have been used for the sizing models validation due to the lack of constructional details.

Results obtained through the application of the presented model have been compared with those coming from a statistical model presented in literature [8] and with available real data. Fig. 7 and Table 10 report the results of the validation process. As it is possible to notice from Fig. 8 the statistical approach seems not to be applicable for high power turbopumps. This confirms that new algorithms (like the one proposed in this paper) are absolutely necessary when dealing with breakthrough innovations and out-of-boundary performance.

The results prove the flexibility of the approach, even if some differences between the computed and the real values still remain. The different results are in line with the statistical model [8] and in the majority of the cases they show better correlation. It is interesting also to see how the estimations seem to be more accurate than the statistical formulation.

It is worth noticing that besides the differences in mass estimation with respect to real data are relevant, the model presented in this paper gives better results in the majority of the cases if compared to the other considered statistical approaches. Of course, it is clear that the next steps in terms of research activities to be performed is the identification of suitable corrective factors properly fitted to take into account the differences in the architectures of the components.

Table 10. Turbopump Database

\begin{tabular}{|c|c|c|c|c|c|c|c|c|c|c|c|c|c|}
\hline కั & 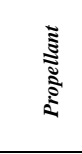 & 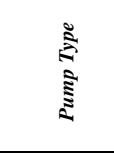 & 气̆ & 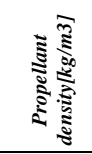 & 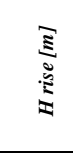 & $\begin{array}{l}\bar{\pi} \\
\stackrel{\bar{\theta}}{y} \\
\bar{y}\end{array}$ & $\begin{array}{l}\bar{n} \\
\stackrel{5}{\Sigma}\end{array}$ & $\begin{array}{l}\overline{\text { जे }} \\
\text { है }\end{array}$ & 胥 & $\Xi$ & 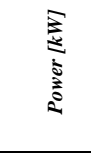 & 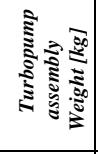 & 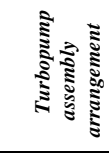 \\
\hline \multirow{2}{*}{ RL10A-3-3 } & LOX & Centrifugal & 1 & 1102.07 & 341.38 & 3.70 & 12.79 & 0,01 & 1267.11 & 62.90 & 70.10 & \multirow[b]{2}{*}{34.52} & \multirow{2}{*}{$\begin{array}{c}\text { Geared O2 } \\
\text { pump }\end{array}$} \\
\hline & LH2 & Centrifugal & 2 & 69.68 & 9692.6 & 6.62 & 2.54 & 0.04 & 3167.77 & 55.00 & 441.45 & & \\
\hline \multirow[b]{2}{*}{$\mathrm{J}-2$} & LOX & Centrifugal & 1 & 1134.11 & 665.99 & 7.41 & 208.84 & 0.18 & 916.61 & 80.00 & 1758.36 & \multirow[b]{2}{*}{303.3} & \multirow{2}{*}{$\begin{array}{c}\text { Dual } \\
\text { Turbopump } \\
\text { series } \\
\text { turbines }\end{array}$} \\
\hline & LH2 & Axial & 7 & 70.48 & 11582 & 8.33 & 37.92 & 0.54 & 2841,05 & 73.00 & 5948.45 & & \\
\hline \multirow[b]{2}{*}{ A-7* } & LOX & Centrifugal & 1 & 1143.72 & 187.76 & 2.11 & 92.99 & 0.08 & 494.07 & 72.00 & 238.62 & \multirow[b]{2}{*}{150.60} & \multirow{2}{*}{$\begin{array}{c}\text { Single } \\
\text { shaft with a } \\
\text { turbine in } \\
\text { the middle }\end{array}$} \\
\hline & Alcohol & Centrifugal & 1 & 906.65 & 347.17 & 2.91 & 68.04 & 0,08 & 494.07 & 70.00 & 311.70 & & \\
\hline \multirow{2}{*}{ MB-3* } & LOX & Centrifugal & 1 & 1143.72 & 503.22 & 5.61 & 206.84 & 0.18 & 660.05 & 79.00 & 1364.63 & \multirow{2}{*}{254.92} & \multirow{2}{*}{$\begin{array}{l}\text { Geared } \\
\text { turbine }\end{array}$} \\
\hline & RJ-1 & Centrifugal & 1 & 852.18 & 712.32 & 5.96 & 91.63 & 0.11 & 660.05 & 72.00 & 902.30 & & \\
\hline \multirow{2}{*}{$\mathrm{H1}^{*}$} & LOX & Centrifugal & 1 & 1134.11 & 564.18 & 6.31 & 243.58 & 0.21 & 699.53 & 77.80 & 1744.94 & \multirow{2}{*}{235.87} & \multirow{2}{*}{$\begin{array}{l}\text { Geared } \\
\text { turbine }\end{array}$} \\
\hline & RP-1 & Centrifugal & 1 & 808.93 & 828,75 & 6.64 & 108.86 & 0.13 & 699.53 & 71.80 & 1245.32 & & \\
\hline \multirow{2}{*}{ MA-5 sustainer* } & LOX & Centrifugal & 1 & 1156.54 & 572.72 & 6.40 & 87.64 & 0.08 & 1063.95 & 64.20 & 759.12 & \multirow{2}{*}{103.87} & \multirow{2}{*}{$\begin{array}{l}\text { Geared } \\
\text { turbine }\end{array}$} \\
\hline & RP-1 & Centrifugal & 1 & 884.22 & 797.36 & 6.34 & 41.55 & 0.05 & 1063.95 & 64.50 & 462.33 & & \\
\hline \multirow{2}{*}{ MA-5 booster } & LOX & Centrifugal & 1 & 1150.13 & 511.76 & 5.70 & 207.75 & 0.18 & 661.20 & 74.30 & 1342.26 & \multirow{2}{*}{396.90} & \multirow{2}{*}{$\begin{array}{l}\text { Geared } \\
\text { turbine }\end{array}$} \\
\hline & RP-1 & Centrifugal & 1 & 812.14 & 665.68 & 5.28 & 95.71 & 0.12 & 661.20 & 73.60 & 858.30 & & \\
\hline F1 & LOX & Centrifugal & 1 & 1161.34 & 943.97 & 10.6 & 1846.15 & 1.59 & 574.70 & 74.60 & 22520.14 & 1428.84 & Single \\
\hline
\end{tabular}




\begin{tabular}{|c|c|c|c|c|c|c|c|c|c|c|c|c|c|}
\hline & RP-1 & Centrifugal & 1 & 810.54 & 1575.2 & 12.5 & 777.92 & 0.96 & 574.70 & 72.60 & 16479.97 & & $\begin{array}{l}\text { shaft with a } \\
\text { turbine ion } \\
\text { end }\end{array}$ \\
\hline \multirow[b]{2}{*}{ LR87-AJ-3* } & LOX & Centrifugal & 1 & 1143.72 & 460.25 & 5.14 & 187.20 & 0.16 & 832.42 & 70.00 & N/A & \multirow{2}{*}{326.59} & \multirow{2}{*}{$\begin{array}{l}\text { Geared } \\
\text { turbine and } \\
\text { pumps }\end{array}$} \\
\hline & RP-1 & Centrifugal & 1 & 808.93 & 878.13 & 6.98 & 83.14 & 0.10 & 919.44 & 70.00 & N/A & & \\
\hline \multirow{2}{*}{ LR91-AJ-3* } & LOX & Centrifugal & 1 & 1143.72 & 491.64 & 5.40 & 79.65 & 0.07 & 936.72 & 70.00 & N/A & \multirow{2}{*}{92.53} & \multirow{2}{*}{$\begin{array}{c}\text { Geared O2 } \\
\text { pump }\end{array}$} \\
\hline & RP-1 & Centrifugal & 1 & 808.93 & 921.72 & 7.27 & 33.61 & 0.04 & 2639.67 & 70.00 & N/A & & \\
\hline \multirow{2}{*}{ YLR81-BA-11* } & IRFNA & Centrifugal & 1 & 1573.02 & 414.53 & 6.38 & 17.83 & 0.01 & 2658.73 & 70.00 & 262.49 & \multirow{2}{*}{27.44} & \multirow{2}{*}{$\begin{array}{l}\text { Geared } \\
\text { turbine and } \\
\text { pumps }\end{array}$} \\
\hline & UDMH & Centrifugal & 1 & 791.31 & 643.13 & 5.00 & 6.94 & 0.01 & 1509.01 & 70.00 & 262.49 & & \\
\hline \multirow{2}{*}{ YLR87-AI-7 } & NPO & Centrifugal & 1 & 1465.69 & 530.35 & 7.57 & 249.48 & 0.17 & 877.76 & 68.00 & 1908.99 & \multirow{2}{*}{219.54} & \multirow{2}{*}{$\begin{array}{c}\text { Geared } \\
\text { NPO pump }\end{array}$} \\
\hline & A-50 & Centrifugal & 1 & 905.05 & 1030.6 & 9.17 & 124.29 & 0.14 & 964.36 & 68.00 & 1849.34 & & \\
\hline \multirow{2}{*}{ YLR91-AJ-7* } & NPO & Mixed flow & 1 & 1473.70 & 522.12 & 7.38 & 93.90 & 0.06 & 880.17 & 67.40 & 715.87 & \multirow{2}{*}{116.12} & \multirow{2}{*}{$\begin{array}{c}\text { Geared O2 } \\
\text { pump }\end{array}$} \\
\hline & $\mathrm{A}-50$ & Mixed flow & 1 & 913.05 & 908.61 & 7.97 & 52.16 & 0.06 & 2480.29 & 57.10 & 812.81 & & \\
\hline SSME-OX HP & LOX & Centrifugal & 2 & 1134 & 62951 & 44.9 & 508 & 0.06 & 3703 & 70.00 & 2785 & 258.75 & $\begin{array}{c}\text { Direct } \\
\text { Driven }\end{array}$ \\
\hline SSME-F HP & LH2 & Centrifugal & 3 & 70.8 & 12601 & 20.4 & 70 & 0.12 & 8378 & 70.00 & 2353 & 348.75 & $\begin{array}{l}\text { Direct } \\
\text { Driven }\end{array}$ \\
\hline Vulcain 1 - Ox & LOX & Centrifugal & 1 & 1134 & 1169 & 13.3 & 208 & 0.18 & 1361 & 70.00 & 5000 & 250 & $\begin{array}{l}\text { Direct } \\
\text { Driven }\end{array}$ \\
\hline Vulcain 2 - Ox & LOX & Centrifugal & 1 & 1134 & 1169 & 13 & 207 & 0.18 & 1361 & 70.00 & 2900 & 185 & $\begin{array}{l}\text { Direct } \\
\text { Driven }\end{array}$ \\
\hline Vinci - Ox & LOX & Centrifugal & 1 & 1134 & 697 & 7.7 & 33 & 0.03 & 1885 & 70.00 & 500 & 49.5 & $\begin{array}{l}\text { Direct } \\
\text { Driven }\end{array}$ \\
\hline Vulcain 2 Fuel & LH2 & Centrifugal & 2 & 70.8 & 14484 & 18 & 45 & 0.63 & 3718 & 70.00 & 14000 & N/A & $\begin{array}{l}\text { Direct } \\
\text { Driven }\end{array}$ \\
\hline Vinci Fuel & LH2 & Centrifugal & 2 & 70.8 & 32777 & 22.4 & 5.8 & 0.07 & 9425 & 76.00 & 2500 & N/A & $\begin{array}{l}\text { Direct } \\
\text { Driven }\end{array}$ \\
\hline
\end{tabular}

*These turbopumps have not been considered for the final mass estimations, because constructional drawings are not available.
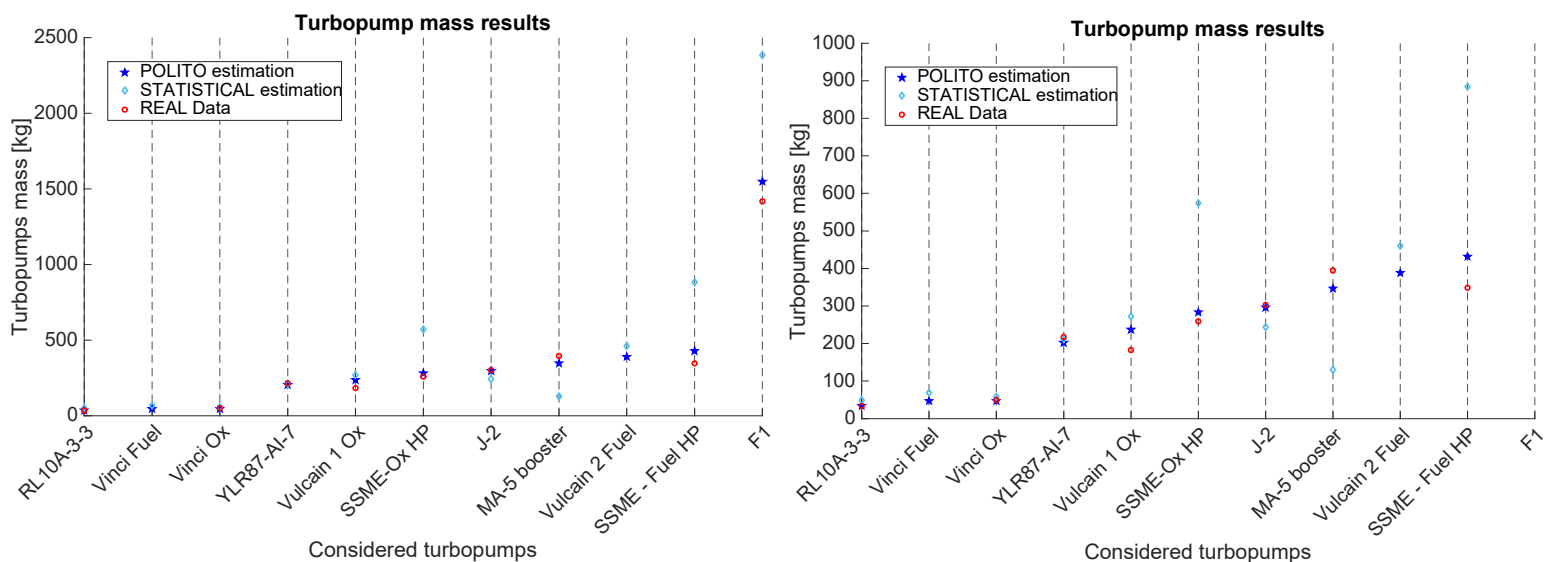

Fig. 7. Summary of turbopumps mass computation and comparison with statistical approach

Table 10. Turbopump analysis results comparison

\begin{tabular}{|l|r|r|r|r|r|}
\hline & $\begin{array}{c}\text { Real Mass } \\
{[\mathbf{k g}]}\end{array}$ & $\begin{array}{c}\text { Mass } \\
\text { POLITO } \\
{[\mathbf{k g}]}\end{array}$ & Mass Stats [kg] & $\begin{array}{c}\text { Error } \\
\text { POLITO } \\
\text { \% }\end{array}$ & $\begin{array}{c}\text { Error } \\
\text { STATS \% }\end{array}$ \\
\hline RL10A-3-3 & 34.25 & 33.28 & 50.26 & $3 \%$ & $47 \%$ \\
\hline Vinci Fuel & N/A & 46.59 & 68.91 & N/A & N/A \\
\hline Vinci Ox & 49.5 & 47.87 & 58.81 & $3 \%$ & $19 \%$ \\
\hline YLR87-AI-7 & 217.8 & 203.16 & 211.44 & $7 \%$ & $-3 \%$ \\
\hline Vulcain 1 Ox & 183.53 & 236.09 & 271.65 & $-29 \%$ & $48 \%$ \\
\hline SSME - Ox HP & 258.75 & 283.35 & 573.42 & $-10 \%$ & $122 \%$ \\
\hline J-2 & 303.3 & 296.67 & 243.51 & $2 \%$ & $-20 \%$ \\
\hline MA-5 booster & 393.75 & 346.12 & 130.50 & $12 \%$ & $-67 \%$ \\
\hline Vulcain 2 Fuel & N/A & 388.01 & 459.03 & N/A & N/A \\
\hline SSME - Fuel HP & 348.75 & 431.48 & 885.03 & $-24 \%$ & $154 \%$ \\
\hline F1 & 1417.5 & 1546.90 & 2386.44 & $-9 \%$ & $68 \%$ \\
\hline
\end{tabular}




\section{LAPCAT Turbopump sizing}

The semi-empirical model presented in the previous sections has been applied to size the turbopump of the LAPCAT MR2. Reference [15] has been a valuable source of inputs for this case study. However, the operational ranges are not the only values required. Indeed, as already reported and discussed in Fig. 6, it is also necessary to make reasonable design assumptions, mainly related to the constructional characteristics of the overall assembly.

Table 11 summarizes both inputs and design assumptions for the LAPCAT MR2. Starting from these operating ranges and assuming an efficiency value of 0.7 , in line with the turbopumps of the considered database, the Cordier diagram has been plotted for different $\mathrm{N}$ values, within the hypothesized range. As previously highlighted, higher $\mathrm{N}$ values move the turbopump towards higher speed number $\sigma$ and lower diameter number $\delta$ (Fig. 8). Considering the ranges of $\sigma$ and $\delta$, it is possible to notice that the points are in the class of radial flow. Thus, a radial configuration is here suggested. Then, different architectures, considering a variable number of stages have been investigated. Eventually, a direct driven turbopump type has been hypothesized. This assumption might be reviewed in future works, where the integration issues of the overall subsystem will be in-depth analysed.

In the following Subsections, the major results for the LAPCAT MR2 vehicle are reported and commented.

Table 11. LAPCAT MR2 - Input and assumptions for turbopump sizing

\begin{tabular}{|c|c|c|c|}
\hline & & $\begin{array}{l}\text { Estimated Value for LAPCAT } \\
\text { MR2 }\end{array}$ & Comments \\
\hline \multirow{5}{*}{ 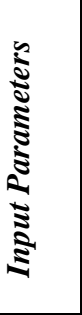 } & Rotational speed $N$ [rpm] & {$[10000-40000]$} & $\begin{array}{l}\text { Considering a typical range of } \\
\text { operations for turbopumps with LH } 2 \\
\text { working fluid. }\end{array}$ \\
\hline & Flow rate $\dot{m}[\mathrm{~kg} / \mathrm{s}]$ & {$[0-100]$} & {$[15]$} \\
\hline & Fluid Density $\rho[\mathrm{kg} / \mathrm{m} 3]$ & 70.8 & Liquid Hydrogen (LH2) \\
\hline & Pressure Rise $\Delta p[\mathrm{~N} / \mathrm{m} 2]$ & {$\left[60 \cdot 10^{5}-80 \cdot 10^{5}\right]$} & {$[15]$} \\
\hline & Efficiency [-] & 0.7 & $\begin{array}{l}\text { Assumption considering currently } \\
\text { available technologies }\end{array}$ \\
\hline \multirow{2}{*}{ 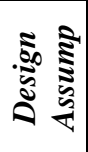 } & Turbopump type & $\begin{array}{l}\text { Radial pump and axial turbine } \\
\text { configuration }\end{array}$ & $\begin{array}{l}\text { Considering the Balje and Cordier } \\
\text { Diagrams }\end{array}$ \\
\hline & Mechanical arrangement & Direct Driven & $\begin{array}{l}\text { Depending on the speed variations } \\
\text { between pump and turbine }\end{array}$ \\
\hline
\end{tabular}

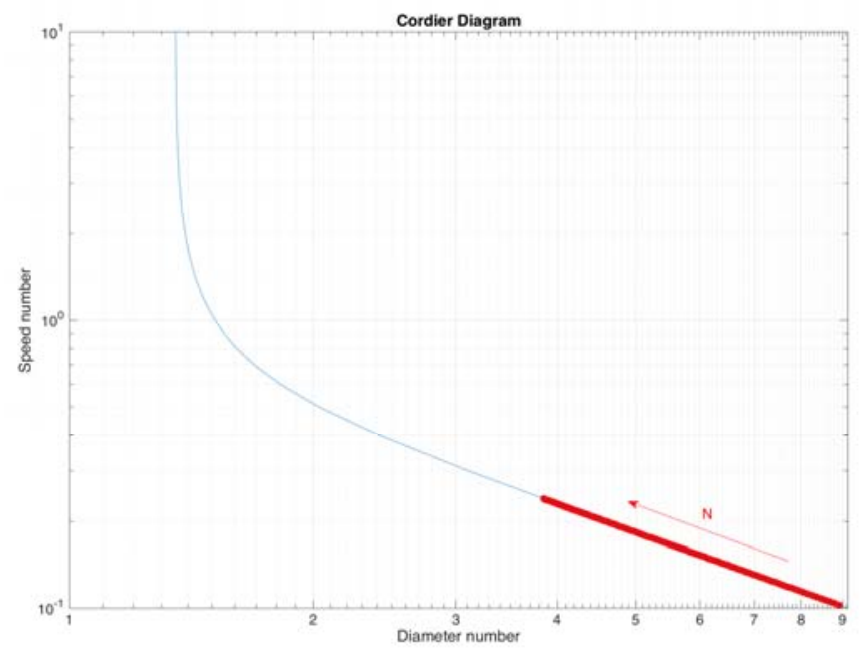

Fig. 8. Cordier Diagram for LAPCAT MR2 turbopump.

Physical characteristics are not subjected to changes during operation, as it happens to the performance, which are dependent to the instantaneous working conditions (e.g. flow rate, pressure drop, power consumption, velocities of the flow etc...). However, the characterization of the machine depends on the selected design point, which will affect the final arrangement of the component in terms of mass and sizing. For this reason, it is crucial to properly select the design point of the considered device not only looking at the performance range, but also referring to its final mechanical configuration (e.g. a certain component may be convenient for the performance but unfeasible because of excessive weight or volume). In this section, the main results of the sensitivity analysis are discussed, with a special focus on the identification of possible trends of physical characteristics with design conditions. Apart from the capability of theoretically predicting the final arrangement of the machine, the analysis of the effects of design 
operating point is one of the crucial advantages of the proposed approach and represents a remarkable added value of this research activity.

\subsection{Sizing example}

Considering the trends reported in the previous subsections, an example of sizing for the turbopump of LAPCAT MR2 has been carried out hypothesizing a high rotational speed $(N=35,000 \mathrm{rpm})$, the highest mass flow $(\dot{m}=$ $\left.100 \frac{\mathrm{kg}}{\mathrm{s}}\right)$ rate and head rise $(\triangle P=80 \mathrm{bar})$ and a single stage impeller and a two-stages turbine in a direct driven

configuration. Moreover, additional assumptions related to more constructional details should be done in order to properly estimate diameter, length and eventually, mass budget.

As far as the fuel pump is concerned, LAPCAT MR2 hypothesized turbopump, a baseline configuration with a single stage pump, with frontal inlet and with an inducer has been envisaged. Moreover, the diffuser will be a volute diffuser, trying to maximize the developed pressure head (in line with Vulcain TP).

These assumptions are collected in Table 12, together with the results.

Table 12. LAPCAT MR2 - outputs of turbopump sizing

\begin{tabular}{|c|c|c|c|c|c|}
\hline & \multirow{2}{*}{\multicolumn{3}{|c|}{ Hypotheses }} & \multirow{3}{*}{ Items } & \multirow[b]{2}{*}{$\begin{array}{l}\text { LAPCAT MR2 } \\
\text { estimated value }\end{array}$} \\
\hline & & & & & \\
\hline & Input & Value & Comment & & \\
\hline \multirow{4}{*}{ Diameter } & $k_{D / d i f f}$ & 1 & Volute type & \multirow{4}{*}{$D_{T P}$} & \multirow{4}{*}{$0.549 \mathrm{~m}$} \\
\hline & $k_{D / \text { inlet }}$ & 0 & $\begin{array}{l}\text { diffuser } \\
\text { Frontal inlet }\end{array}$ & & \\
\hline & $\boldsymbol{k}_{D / \text { outlet }}$ & 0 & Not applicable & & \\
\hline & $k_{D / m i s c}$ & 0.01 & Compact design & & \\
\hline \multirow{4}{*}{ Length } & $k_{L / \text { ind }}$ & 2.5 & Inducer & \multirow{4}{*}{$L_{T P}$} & \multirow{4}{*}{$0.8734 \mathrm{~m}$} \\
\hline & $\boldsymbol{k}_{L / p \boldsymbol{b}}$ & 0 & & & \\
\hline & $k_{L / o u t}$ & 1 & Mean of the case- & & \\
\hline & $\boldsymbol{k}_{\text {L/other }}$ & & Compact design & & \\
\hline \multirow[b]{2}{*}{ Mass } & $I_{N}$ & 0.6 & $\begin{array}{c}\text { Considering } \\
\mathrm{N}=35000 \mathrm{rpm}\end{array}$ & \multirow[b]{2}{*}{$M_{T P}$} & \multirow[b]{2}{*}{$456 \mathrm{~kg}$} \\
\hline & $I_{\dot{m}}$ & 1 & $\begin{array}{l}\text { Considering } \\
\text { a flow rate of } 100 \\
\mathrm{~kg} / \mathrm{s}\end{array}$ & & \\
\hline
\end{tabular}

Table 13. LAPCAT MR2 -sensitivity analysis on turbopump sizing

\begin{tabular}{|c|c|c|c|}
\hline & $\begin{array}{c}1 \mathrm{TP} \text { of } \\
100 \mathrm{~kg} / \mathrm{s}\end{array}$ & $\begin{array}{c}\mathbf{2} \mathrm{TPs} \text { of } \\
\mathbf{5 0} \mathrm{kg} / \mathrm{s}\end{array}$ & $\begin{array}{c}\mathbf{4} \mathrm{TPs} \text { of } \\
\mathbf{2 5} \mathrm{kg} / \mathbf{s}\end{array}$ \\
\hline $\begin{array}{c}\text { Mass of a single } \\
\text { Turbopump }\end{array}$ & 456.7 & 449.1462 & 447.23 \\
\hline $\begin{array}{c}\text { Mass of the overall } \\
\text { assembly }\end{array}$ & 456.7 & 898.29 & 1789 \\
\hline
\end{tabular}

It is worth noticing that $100 \mathrm{~kg} / \mathrm{s}$ is the maximum flow rate that LAPCAT MR2 will need during a typical mission. However, this happens in a very specific condition, while during cruise, the required mass flow rate of liquid hydrogen is about $20 \mathrm{~kg} / \mathrm{s}$. Thus, one would not opt for one single pump for different reasons. Redundancy is a key driver to split the pump unit into different smaller ones. These can be placed optimally at the lowest points of the various tanks on-board while being also interconnected. The pump size can be tuned not only to the present state-of-the- 
art but also serve the need of redundancy and avoidance of single-point failure. This is an element which still needs to be worked out in the future to assure a workable solution from a pure safe operational point of view. Table 13 reveals that, hypothesizing a constant eta coefficient $(\eta=0.7)$, the exploitation of a higher number of turbopumps to provide the requested mass flow, is not convenient in terms of mass increase. However, the exploitation of two turbopumps, instead of a single one, may bring noticeable benefits from the safety standpoint, although a non-negligible mass increase. The assumption of a constant efficiency coefficient will be relaxed in future, when optimization cycles will be performed in order to re-evaluate the ideal eta coefficient of the different turbomachinery in the various mission phases.

\section{Conclusions}

Major goal of this paper was the development of a general methodology to support the sizing of innovative Thermal Control Subsystems for high-speed transportation systems. This methodology should be sufficiently general to be exploited for the derivation of Estimation Relationships (ERs) of sizing characteristics as well as mass, volume and power budgets both for active and passive components. Following the suggested approach, ad-hoc semi-empirical models relating the geometrical sizing, mass, volume and power features of each component to operating conditions have been derived starting from an in-depth literature review activity. At the beginning of this paper, a general methodology to tackle the highlighted problems has been proposed and then it has been applied to the derivation of a semi empirical parametric model for turbopumps sizing.

For the sake of clarity, a real test case has been considered. The overall turbopumps sizing algorithms have been applied to the Thermal and Energy Management Subsystem for the LAPCAT MR2 vehicle as example of highly integrated multifunctional subsystem. The estimations led to a turbopump of noticeable dimensions but it has to be underlined that these results have been derived on the basis of some conservative assumptions. Indeed, in future, a review of the presented algorithms, both in its formulation and in the suggested values for the parametric coefficients will be performed. This activity will allow to consider future productive scenarios where new available materials or technologies could be implied.

\section{References}

[1] J. Steelant and T. Langener, 'The LAPCAT-MR2 Hypersonic Cruiser Concept', ICAS-2014-0428, 29th Congress of the International Council of the Aeronautical Sciences, St. Petersburg, September 7-12, 2014.

[2] V. Fernandez Villace and J. Steelant. The Thermal Paradox of Hypersonic Cruisers. International Space Planes and Hypersonic Systems and Technologies Conference. 6-9 July 2015, Glasgow, Scotland.

[3] J. Steelant, R. Varvill, S. Defoort, K. Hannemann and M. Marini. Achievements Obtained for Sustained Hypersonic Flight within LAPCAT-II Project. International Space Planes and Hypersonic Systems and Technologies Conference. 6-9 July 2015, Glasgow, Scotland.

[4] Roncioni P., Natale P., Marini M., Langener T. and Steelant J., 'Numerical Simulations and Performance Assessment of a Scramjet Powered Cruise Vehicle at Mach 8', Journal of Aerospace Science and Technology, Vol 42, p 218-228, Jan. 2015, DOI 10.1016/j.ast.2015.01.006

[5] Fernández-Villacá V., Paniaguá G. and Steelant J., 'Installed Performance Evaluation of an Air Turbo-Rocket Expander Engine', J. of Aerospace Science and Technology, Vol. 35, pp 63-79, 2014, DOI:10.1016 /j.ast.2014.03.005.

[6] Meerts C. and Steelant J., 'Air Intake Design for the Acceleration Propulsion Unit of the LAPCAT-MR2 Hypersonic Aircraft', 5th European Conference for Aeronautics and Space Sciences (EUCASS), Munich, Germany, 1-5 July 2013.

[7] T. Langener, S. Erb and J. Steelant. Trajectory Simulation and Optimization of the LAPCAT-MR2 Hypersonic Cruiser Concept. $29^{\text {th }}$ Congress of the International Council of the Aeronautical Sciences. 7-12 September 2014, St. Petersburg, Russia.

[8] A. J. Sobin. Turbopump Systems for Liquid Rocket Engines. NASA Space Vehicle Design Criteria SP-8107 (1974)

[9] W. E. Campbell and J. Farquhar. Centrifugal Pumps for Rocket Engines. NERVA Rocket Operations (1974)

[10]D. K. Huzel and D. H. Huang. Design of Liquid Propellant Rocket Engines. Rocketdyne Division North America Aviation Inc. (1967)

[11]P. Epple, F. Durst and A. Delgado. A theoretical derivation of the Cordier diagram for turbomachines. Proceedings of the IMechE Vol. 225 Part C: Journal of Mechanical Engineering Science (2010).

[12]D. J. Saunders. A method of calculating the weight and dimensions of a turbopump for rocket propellants. Technical note of the Royal Aircraft Establishment (1979)

[13]A.S. Rangwala. Turbo-Machinery Dynamics: design and operations. Mechanical Engineering Series, McGraw-Hill, 2005.

[14] J. Steelant and M. van Duijn. Structural Analysis of the LAPCAT-MR2 Waverider Based Vehicle. 17 ${ }^{\text {th }}$ AIAA International Space Planes and Hypersonic Systems and Technology Conference. 11-14 April 2011, San Francisco, California 
[15] S. Balland, V. Fernandez Villace and J. Steelant. Thermal and Energy Management for Hypersonic Cruise Vehicles - Cycle Analysis. International Space Planes and Hypersonic Systems and Technologies Conference. 6-9 July 2015, Glasgow, Scotland.

[16] T. J. Hart. Design Criteria and Analyses for Thin-Walled Pressurized Vessels and Interstage Structures 